\title{
Task and Context Sensitive Gripper Design Learning Using Dynamic Grasp Simulation
}

\author{
A. Wolniakowski (i) - K. Miatliuk - Z. Gosiewski • L. Bodenhagen • \\ H. G. Petersen • L. C. M. W. Schwartz • J. A. Jørgensen • \\ L.-P. Ellekilde $\cdot$ N. Krüger
}

Received: 19 October 2015 / Accepted: 18 January 2017 / Published online: 25 March 2017

(C) The Author(s) 2017. This article is published with open access at Springerlink.com

\begin{abstract}
In this work, we present a generic approach to optimize the design of a parametrized robot gripper including both selected gripper mechanism parameters, and parameters of the finger geometry. We suggest six gripper quality indices that indicate different aspects of the performance of a gripper given a CAD model of an object and a task description. These quality indices are then used to learn task-specific finger designs based on dynamic simulation. We demonstrate our gripper optimization on a parallel finger type gripper described by twelve parameters. We furthermore present a parametrization of the grasping task and context, which is essential as an input to the computation of gripper performance. We exemplify important aspects of the indices by looking at their performance on subsets of the parameter space by discussing the decoupling of parameters and show optimization results for two use cases for different task contexts. We provide a qualitative evaluation of the obtained results based on existing design guidelines and our engineering experience. In addition, we show
\end{abstract}

A. Wolniakowski $(\bowtie) \cdot$ K. Miatliuk $\cdot$ Z. Gosiewski

Automation and Robotics Department, Białystok

University of Technology, Białystok, Poland

e-mail: adam.wolniakowski@gmail.com

L. Bodenhagen - H. G. Petersen - L. C. M. W. Schwartz •

J. A. Jørgensen · L.-P. Ellekilde · N. Krüger

The Maersk Mc-Kinney Moller Institute, Faculty

of Engineering, University of Southern Denmark,

Odense, Denmark that with our method we achieve superior alignment properties compared to a naive approach with a cutout based on the "inverse of an object". Furthermore, we provide an experimental evaluation of our proposed method by verifying the simulated grasp outcomes through a real-world experiment.

Keywords Gripper design · Industrial assembly • Simulation · Optimization

\section{Introduction}

The successful execution of grasping in a robotics system is essential in industrial applications where grasp failure can result in anything from an expensive reduction in throughput to the destruction of parts or fabrication hardware. With the gripper being the main tool that physically interacts with the environment, it is obvious that its design characteristics are important for successful grasping. Moreover, robot systems that rely on sensors for object detection and pose estimation introduce significant uncertainties in the system, that will influence grasp success and thereby add additional demands to the robustness of the gripper design. Under these constraints, gripper design can be seen as a demanding task.

When switching the context in industrial processes, it is required to quickly adapt the existing robot setup to handle a new set of tasks. In case of commonly used parallel finger grippers, the driving mechanism (or the 
Fig. 1 Two important parts of the gripper design problem. The kinematic design (bottom) is typically determined from off-theshelf products whereas the gripper jaw design (top) is customized to allow better grasping of one or multiple specific objects

\section{Gripper Design}

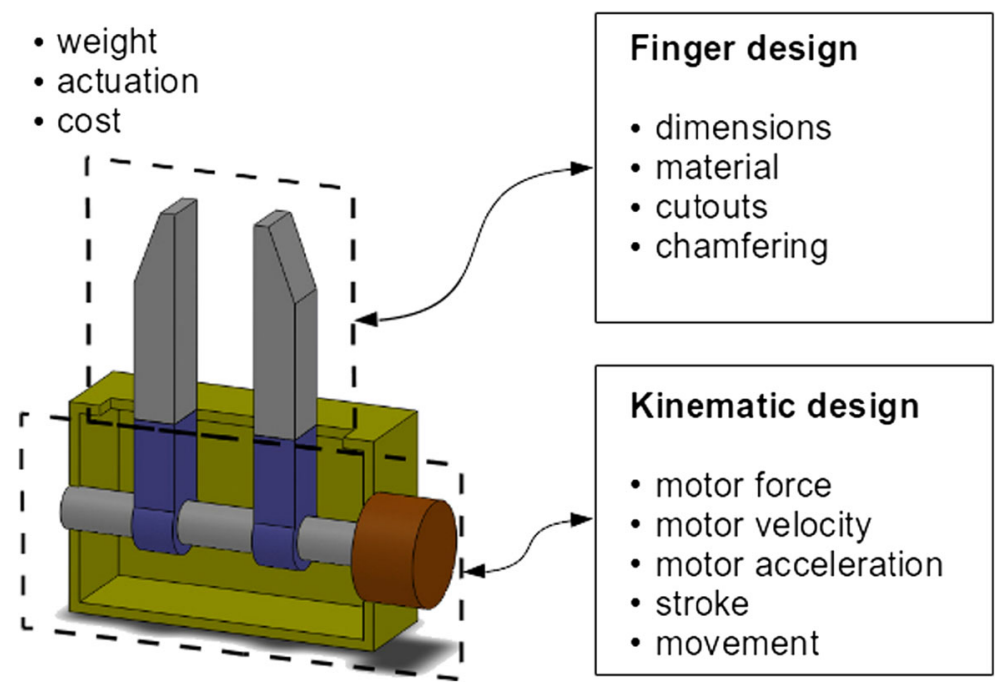

base) would be determined from off-the-shelf products, whereas the gripper jaw would have to be specifically designed to handle new objects (see Fig. 1). An example of a robotic setup with fixed base and interchangeable fingers can be seen in Fig. 2. Using off-the-shelf components is important for non-expert floor operators, since it enables them to maintain and modify the robot cell. This is especially crucial in an industrial context, where SMEs ${ }^{1}$ cannot rely on expensive experts to reprogram their robots in rapidly changing production facilities. Still, the task of designing the gripper fingers can introduce severe delays in the context change and often requires a large degree of engineering expertise. Therefore, it would be beneficial to have a method for automizing the task of finding an appropriate gripper design for specific contexts.

As can be seen in Fig. 1, the design of a gripper can be complex and any sub-aspect will contain a certain amount of parameters. We split the gripper learning problem into three main tasks:

1. The selection of gripper design parametrization which could include kinematic layout and finger surface design.

2. The efficient and accurate evaluation of a specific gripper parametrization.

3. An efficient search in the high dimensional spaces spanned by the gripper parametrization.

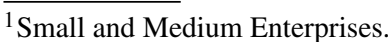

In this paper, we propose a system for the automatic computation of the optimal gripper design for a specific task and context - that is addressing these three problems. The method is based on dynamic simulation of the performance of a gripper. The simulation can be fed with predefined descriptions of off-the shelf gripper components that influence performance through parameters such as stroke, motor force, number of fingers etc.

The approach proposed in this paper can also be viewed as an alternative to dexterous grasping: instead of considering a complex hardware device being able to realize a large variety of grasps, we will design an algorithm to compute the gripper design for specific tasks. Doing that, slow, expensive and still rather unstable dexterous grippers are avoided and fast and inexpensive hardware can be used. Clearly, this approach will be less flexible considering the time it will take to change the grasp context or the object, which would require computing new grippers. However, in industrial applications, context switches are usually required in periods of days or weeks and not seconds or minutes as in the many frequently changing manipulation tasks that humans usually perform. We would like to stress that our proposed solution is intended to be applied in industrial contexts only, while in other domains (e.g. assistance robotics) grippers with higher degree of dexterity would be of advantage.

An overview of our approach is presented in Fig. 3. Based on the grasping context (Fig. 3a), including 
Fig. 2 A typical robot gripper setup. a - an UR robot is used to pick up a metal box. $\mathbf{b}$ - a closer look at the printed gripper fingers
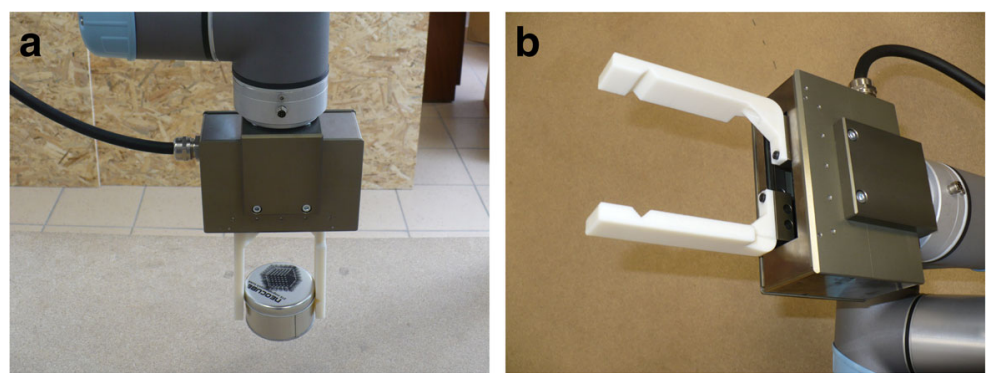

general constraints on the gripper type, the object geometry, and the task description, we can generate gripper designs and test their performance using dynamic simulation or - eventually - direct experimentation (Fig. 3b). The obtained data is collected and can be used for gripper design validation and optimization.

The main contributions of this paper are to introduce:

- a gripper evaluation method based on dynamic grasp simulation, with gripper quality indices which include robustness toward uncertainties in the real world setup.

- a system aimed at optimizing the gripper design for specific tasks, using that gripper evaluation.

- a parametrization of the gripper, and the context, that allows for the inclusion of task constraints during the process of the gripper optimization.
- a gripper optimization strategy utilizing a downhill simplex method search [1] in this highdimensional parameter space.

This paper is based on two conference papers, one on gripper evaluation by means of an objective function [2] and the other on the learning of the gripper shape by optimizing this objective function through gradient descent [3]. Compared to [2] and [3], in this paper we present:

- Additional experiments with 2 objects and 3 task contexts with multiple approach directions.

- The extension of the parametrization of the gripper finger shape, and the extension of the objective function now considering the important aspect of object alignment.

- An analysis of the 12 dimensional objective function in terms of its mathematical properties.
Fig. 3 System overview: a - the input including the description of the grasped object and the context is provided. $\mathbf{b}$ - the simulation is used as a tool to evaluate and optimize proposed gripper designs. Evaluation results and obtained designs are stored for further use

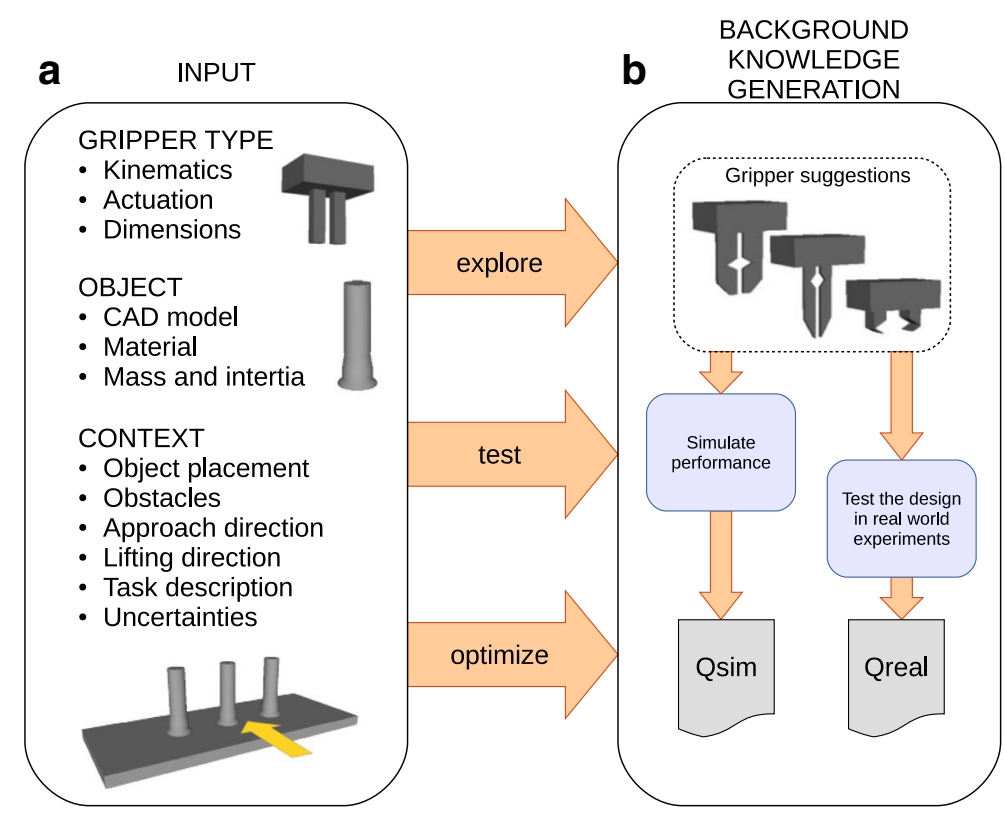


- An extended state of the art section and discussion of future work.

- A real-world experiment to prove the validity of using simulation to predict gripper performance.

The paper is organized as follows: in Section 2, we provide a survey of currently utilized state-of-theart gripper design methods and discuss related work done in the field. Then in Section 3, we present the system overview, in which we describe the general idea, and the tools used: simulation, grasp planning, and the optimization method (Section 4). Then, we provide a detailed description of introduced gripper quality indices. In Section 5, we describe the experiments done in simulation and in the real-world setting, introducing our experimental setup, the proposed task description, and the gripper parametrization. Finally, we discuss the obtained results, and provide a short summary in the conclusion (Section 6).

\section{Related Work}

The problem of gripper design has gathered interest of many researchers and enterprises, resulting in a number of publications on the subject. The research on the topic focuses on four important areas: 1) qualitative gripper and finger shape design guidelines, 2) attempts to quantify gripper performance qualities using analytical methods, 3) the evaluation of grasp performance in simulation as well as 4) computing gripper design parameters by optimization.

Qualitative Gripper Design Guidelines The difficulty of designing robot grippers has motivated the formulation of several gripper design guidelines [4-6]. One of the difficulties implied in these works is that design objectives may conflict, e.g., having a design which is both light and rigid. The design objectives include amongst others: small gripper footprint, small weight, secure grasping, small finger length, avoiding tool changes and aligning grasped objects. Furthermore, reviews on the gripper design problem are presented in [7, 8]. In [7] a general overview of early work on gripper designs and control is presented, whereas grippers designed specifically for handling fruits were presented in [8].

In the thesis presented at the Case Western Reserve University [9], gripper design guidelines were formulated and classified into three groups which contain guidelines to

1) increase system throughput, i.e. minimize interference, minimize weight, ensure a secure grasp of the part, etc.;

2) increase system reliability, such as minimize finger length, design the necessary approach clearance, and others; and

3) reduce gripper cost, such as use less expensive parallel jaws actuators, use off-the-shelf components for designing the gripping system, favour designs which handle multiple parts with a single gripper.

Following these finger design guidelines enables an experienced engineer to manually design an optimal gripper solution for many of the industrial problems. In this paper, we express aspects of these qualitative guidelines in our indices that describe different aspects of grasp quality in a quantitative way.

Quantitative Evaluation of Gripper Designs A substantial amount of scientific work on optimal gripper design is based on kinematic evaluation, especially of the gripping mechanism. Early work on the evaluation of gripper mechanism was based on Merit indices that described the mechanical effectiveness (Grasp Index G.I.) of a gripper [10] and the Capability Index (C.I.), the latter describing the capability of a gripper for grasping objects of varied dimensions. In [11] these Merit indices are used in the optimization of the kinematic design of a gripper. The Merit indices are fast to compute but they are limited to kinematic evaluations and cannot distinguish between changes in the gripper jaw surface.

In the work of J. Cuadrado and colleagues [12] some properties of the gripping mechanisms have been considered to deduce a useful analytical formulation. The synthesis problem has been formulated as an optimization problem over the dimensions of the gripper mechanism linkage (dimensional design), without considering the task context or dynamic parameters beside grasp force.

Ceccarelli et al. in [13] worked on the problem of the design of different kinds of gripper mechanisms. In their work, the synthesis of gripper mechanisms is performed using Cartesian coordinates. The formulation of optimum synthesis task was based on practical design requirements and the aim was to derive an analytical formulation using an index of performance 
(Grasping Index) to describe both kinematic and static characteristics.

Changing the geometry of gripper fingers can improve how well a gripper aligns objects during grasping. Aligning objects enables more secure grasps and by that enables more accurate placement. Gripper jaw design for object alignment was investigated in [14-17]. In [16] they define a modular gripper surface based on trapezoidal segments for which they present an algorithm that can optimize the gripper design such that a specific alignment of the object is obtained when it is grasped from the top.

In [18] the overall objective function is formulated as a combination of two objective functions. The first objective function is written as the range of gripping forces for the assumed gripper stroke. The second is the force transmission ratio which is the ratio between the applied actuating force and the resulting minimum gripping force.

In our work we introduce a new set of quality indices, which express the performance of the gripper based on the statistical analysis of the results of simulation, while taking into account the context information.

Gripper Evaluation Using Simulation Some research on gripper design uses simulation - either in material strength sense, or to test the gripper performance. In [19] a theoretical analysis coupled with simulationbased verification aimed at justifying a reconfigurable Robot Gripper System (RGS), which is used to handle limp material. The design parameters of the RGS mechanism are defined as follows: length, diameter, payload of each arm, maximum stress and deflections as well as natural and damped frequency of each arm.

The statement, that in order to find a useful grasp both the object itself and the task should be considered, is confirmed by Song et al. in [20]. They consider object categories together with task constraints as variables in the Bayesian network, from which full hand configuration can be derived. All experiments are done in simulation with a human hand model.

The simulation based method is also applied by E. Nikandrova and V. Kyrki [21] to find an optimal grasp. A probabilistic approach for task-specific stable grasping of objects with shape variations inside the category is presented in the work. An optimal grasp is found here as a grasp, that is most likely to be task compatible and stable taking into account shape uncertainty in a probabilistic context.
The work in [17] presents a semi-automatic design of gripper jaws for aligning objects. The authors use molding and a convex-hull based method of generating a finger geometry for a specific object, and present a comparison of gripper aligning performance in realworld and simulation based experiments, showing that the latter can accurately reflect the former.

Another use of dynamic simulation was presented in [22], where the kinematic design of an underactuated 2 finger gripper was optimized by first generating a database of grasps using simulation with a fully actuated gripper, which secondly was used to optimize the under-actuated gripper such that it would be able to execute the same grasps as defined in the grasp database. Our use of simulation is similar. However, we define statistical gripper metrics that are computed based on generated grasp databases which then can be used to compare the performance between grippers.

Computing Gripper Design Parameters by Optimization In many works, the task of feasible gripper selection is reduced to an optimization problem which uses an analytical formulation of the gripping mechanisms parameters. In general, a suitable algorithm is developed for an optimal synthesis or selection of gripping mechanisms using various gripper quality criteria.

The robotic gripper design problem described in [23] is based on twenty nine design parameters. The design parameters formally represent physical or functional attributes of the designed robot gripper and the different combinations of their values distinguish the alternative designs. The design task involves a decision-making procedure regarding kinematic and geometric aspects such as function, structure, configuration, material and geometry of the designed gripper. Optimal design parameters are found through the use of a genetic algorithm (GA), where each gene represents a gripper concept.

GA is also used in [24] for optimizing multiple criteria of the kinematic design of spherical serial mechanisms. Conceptual design, fuzzy set methods and mechatronic indices are all used in the mechatronic design of robot grippers for handling fabrics in [25]. Grasping performance quality characteristics, such as wrench space quality measure and robustness measure are described and explored in [26] where the focus is on automatic grasp generation and learning for industrial bin-picking. 
The approach to design optimization of robot gripper proposed by Krenich [27] also presents a force transmission ratio and in addition a displacement transmission ratio as optimization criteria. To generate the optimal solutions, three algorithms are applied - a gradient based method, a random search method and an evolutionary algorithm.

Recently, a new eGrip online tool [28] for designing the optimal gripper shape has been presented by the Schunk company. The tool allows the user to generate a form-fit finger geometry obtained by using a molding method with a provided object CAD model. This method only takes the task context and grasping uncertainties into account to a limited degree, and is best suited for designing single-purpose grippers. Thus it cannot completely replace the required expertise in gripper design.

The method proposed in this paper is not as fast to compute as already existing methods but is instead more generic, accurate and enables the inclusion of context. This is mainly achieved by relying on evaluating grasps using a dynamic grasp simulator. Such a tool can easily include large parts of the task context in the evaluation of the gripper, which more accurately captures the actual task in which the gripper is to be used. Furthermore, accuracy over kinematic simulations is also due to a greater number of modeling parameters such as friction and motor control.

To summarize, our work defines suitable gripper qualities based on evaluating a set of feasible grasps. Compared to previous work, we pursue a statistical approach relying heavily on dynamic simulation, grasp quality metrics and we include contextual information in the evaluation of the gripper. The inclusion of context was demonstrated to be of importance in [29] when evaluating grasp quality. For computing optimal gripper designs, we believe that context is equally important and therefore our method also relies on a specification of the context - namely the task description. The context strongly influences how an object can be grasped, for example a structured context may require much less coverage than an unstructured one due to variation in object poses. Hence, by including context into the evaluation of a gripper, we are able to increase the accuracy of the gripper evaluation and we also believe that quality can be defined in more user intuitive terms that relate to the actual task requirements.

\section{System Overview}

In this section, we provide a detailed overview of our proposed system that evaluates and optimizes gripper designs (see Fig. 3). The Background Knowledge Generation is a component responsible for evaluating gripper designs in the context of objects to be grasped and the task. The evaluation can be performed either through simulation, or alternatively via realworld experiments. However in this paper we focus on the simulation-based evaluation. Obtained knowledge about gripper performance can subsequently be used to explore the gripper parametrization space, and to optimize the proposed designs.

In the following subsections, we will explain the proposed method for gripper evaluation (Sections 3.13.3). Furthermore we will describe the optimization component of the system (Section 3.4).

The gripper evaluation relies on dynamic grasp simulation to compute a set of gripper quality indices (explained in detail in Section 4). For the given gripper design, the object, and the task description, multiple grasps are sampled, generated, and simulated (see Fig. 4c). The indices represent several important aspects of gripper performance (including: success, versatility, alignment, ...- see Fig. 4d), and are based on collecting statistics from the simulation results. The evaluation method overview is shown in Fig. 4, where the inputs (encapsulated in Fig. 4a) are an object model, a gripper design and a task description. Then, we generate a database of grasp targets (Fig. 4b), which is used for simulation, and to determine the grasp coverage of the object for the specific gripper design. The grasp planner is further described in Section 3.1 below. After that, we simulate a subset of generated grasps (see Fig. 4c and Section 3.2), and collect the simulation results, which we then use to compute gripper quality indices (Fig. 4d). This step is explained in detail in Section 3.3. The composition of the grasp sets during the grasp generation and evaluation steps is presented in Fig. 5. Finally, the output of our evaluation method is a set of gripper quality indices (Fig. 4e), explained in Section 4.

\subsection{Grasp Generation}

The first step in our method (see Fig. 4b) is to generate a set of grasps that with a reasonable resolution 


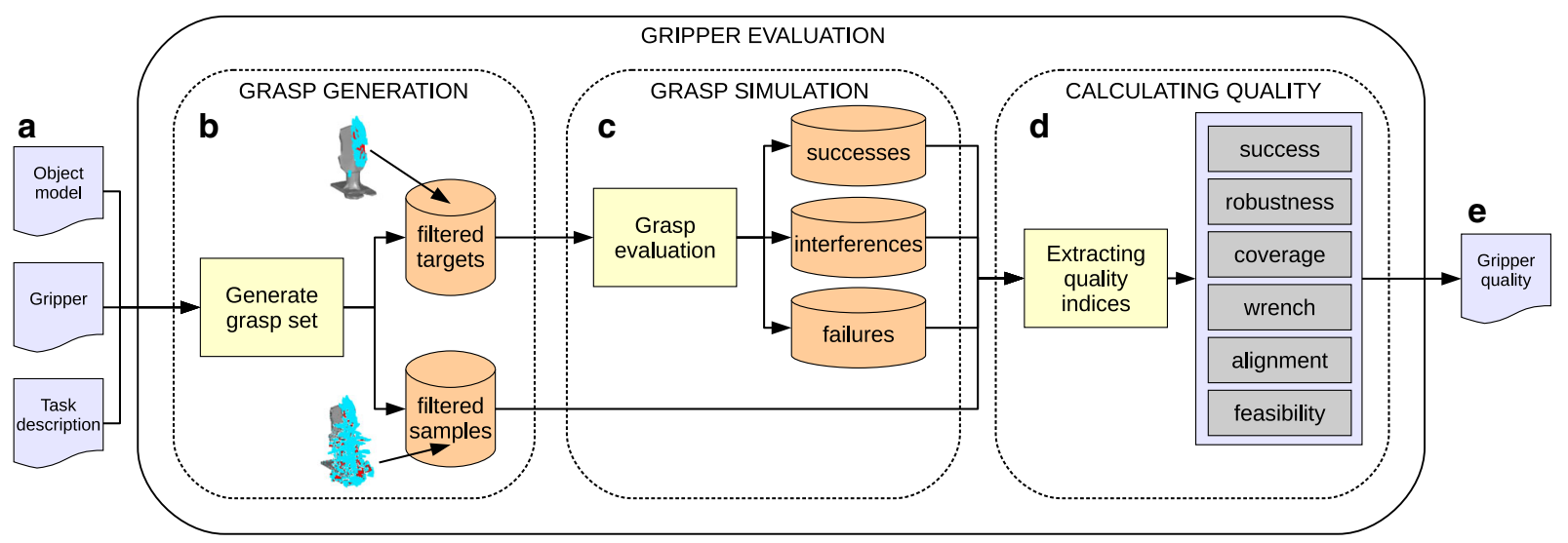

Fig. 4 The overview of the gripper evaluation process

covers all possible grasps of the object within the task description. In our case of parallel finger grippers, this computation is essentially a sample based grasp planner that bases the sampling on the presence of nearly parallel surfaces in the target object's geometry. This sampling heuristic is based on the method described in [29]. We want to stress that the quality indices we propose are generic and can also be applied to other grippers. However, when evaluating other types of grippers, the initial sampling strategy would need to be adapted, to generate a proper grasp set for those grippers.

The grasp planning is done in three steps (see the top part of the Fig. $5 \mathrm{a}-\mathrm{c})$ :

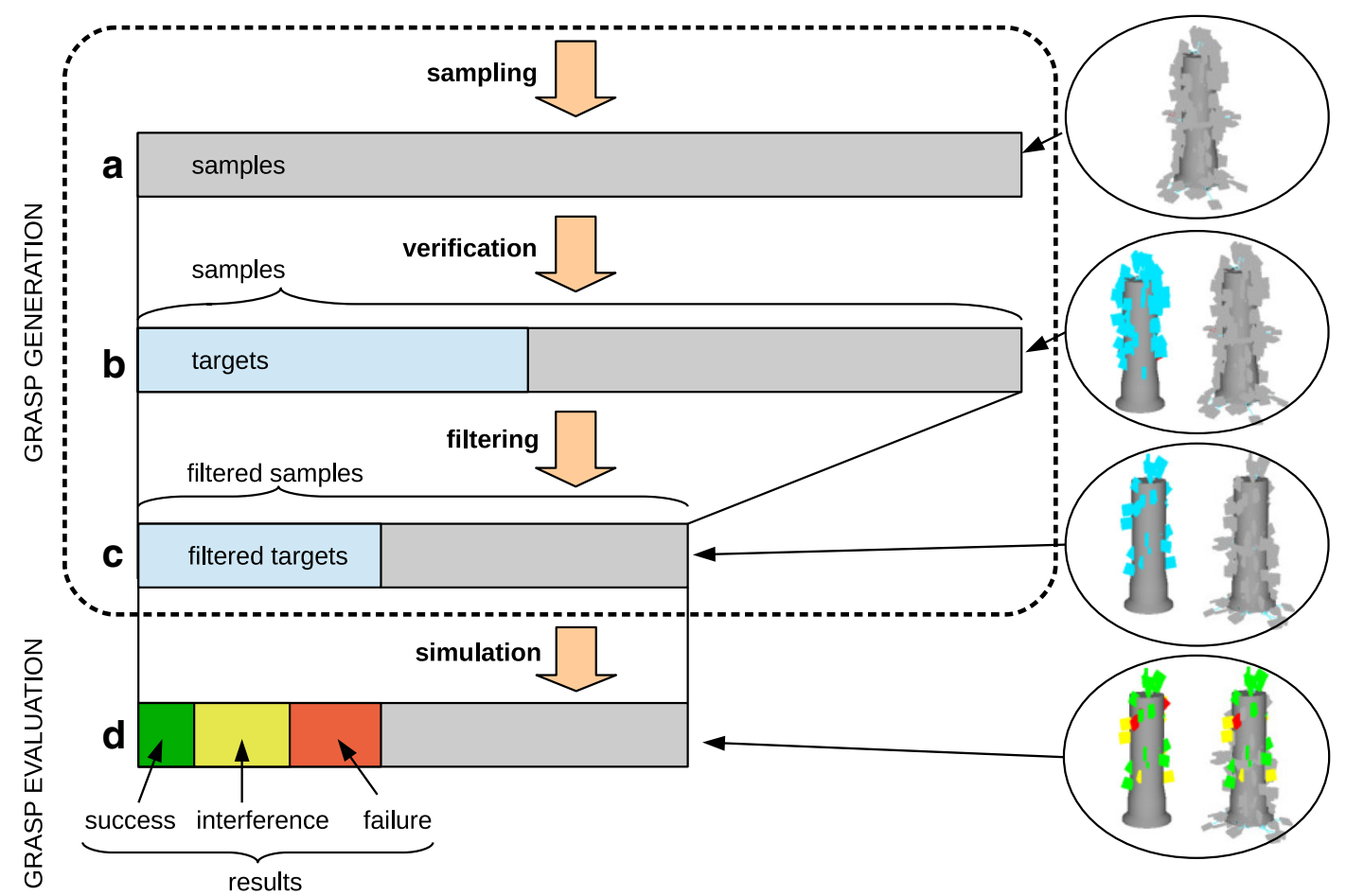

Fig. 5 The breakdown of grasp sets during the steps of the grasp planning and the grasp evaluation. On the right: the corresponding distribution of grasp targets, samples, and results based on the simulation outcome (observe color coding) 
1. Grasp sampling: The grasp candidates are sampled using a heuristic that calculates grasps based on surface point pairs with close to opposite surface normals. We define the set of grasps obtained from the grasp sampling step as the samples set (see Fig. 5a).

2. Grasp verification: The samples are verified such as to reduce the grasp set by removing grasps that would cause collision with the object initially, and thus should be disregarded in the simulation. We define the subset of the grasps that passed the verification step as the set of targets (see Fig. 5b).

3. Grasp filtering: The targets and the samples are filtered such as to avoid clustering in the density of grasp targets over $S E 3$. Thus we obtain the sets of filtered targets and filtered samples (see Fig. 5c).

The grasps within the set of filtered targets are subsequently evaluated using dynamic simulation (see Section 3.2), while the set of filtered samples is retained for use in calculating the gripper coverage index (see Section 4.3).

We provide a detailed description of sampling, verification, and filtering steps of the grasp generation process in Sections 3.1.1-3.1.3 below.

\subsubsection{Grasp Sampling}

Let us assume that the parallel gripper has a defined TCP frame which is placed in-between the fingers with the $z$-axis pointing in the approach direction and the $x$-axis pointing in the direction of the finger movement. Furthermore, let us assume that the approximate minimum and maximum distances $\left(d_{\text {closed }}, d_{\text {open }}\right)$ between the gripper fingers are defined.

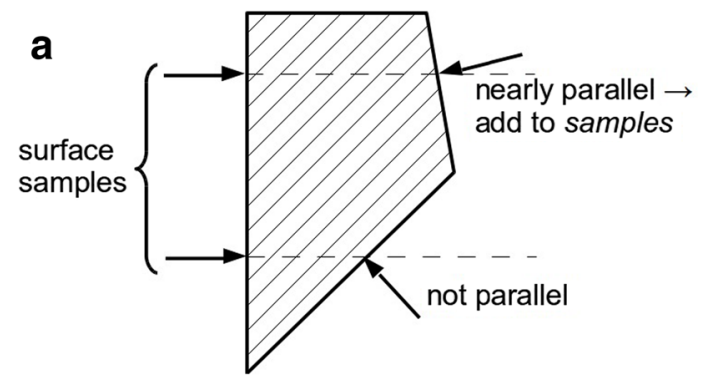

Points on the surface of the target object mesh are uniformly sampled using a cumulative distribution function over the surface area of the mesh. Each sampled surface point is a tuple $\{p, n\}$ containing the position and the surface normal. The method creates a set of surface samples and exhaustively computes all features $\left(2 \times 2\right.$ tuples $\left.\left\{\left\{p_{i}, n_{i}\right\},\left\{p_{j}, n_{j}\right\}\right\}\right)$, where the angle between $n_{i}$ and $n_{j}$ is in the interval $\left[130^{\circ} ; 230^{\circ}\right]$ and where the distance $\left\|p_{j}-p_{i}\right\|$ is in the interval $\left[d_{\text {closed }} ; d_{\text {open }}\right]$. The requirement for two opposite surfaces being nearly parallel for the sampling process is illustrated in Fig. 6a.

For each feature, a grasping pose $T_{\text {target }}^{o b j}$ described relative to the object is calculated. The position part of the pose is calculated as follows:

$P_{\text {target }}^{\text {obj }}=p_{i}+\left(p_{j}-p_{i}\right) / 2$

The orientation is defined by first placing the $x$ axis of $T_{\text {target }}^{o b j}$ along $\left(p_{j}-p_{i}\right) /\left\|p_{j}-p_{i}\right\|$ after which the $z$-axis is rotated by a random angle around the $x$ axis. All of these randomly sampled poses are added to the samples set. The distribution of the samples for a typical object geometry is presented in Fig. 5a.

\subsubsection{Grasp Verification}

It is not necessary to simulate all the grasps stored in the samples set, because many of them can be evaluated kinematically by checking if placing the gripper in the sample configuration would result in an initial collision with elements of the workcell. Thus, we do grasp verification to prune the samples set to extract the grasp targets using the following method: For each of the poses stored in the samples set, the gripper is

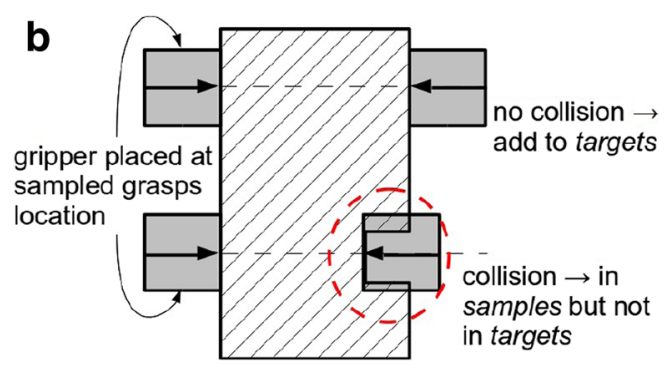

Fig. 6 a-Generation of samples. Mesh surface is sampled for points which provide approximately parallel opposite surfaces, which are not too far apart. b - Filtering of samples to exclude grasps which generate collision initially 


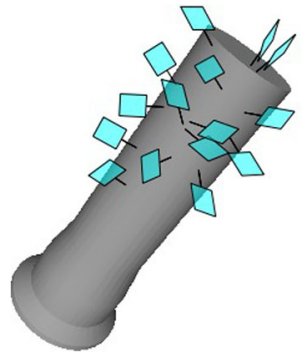

Fig. 7 Filtering of the samples to keep the database uniform in $S E 3$. It is undesirable to have the targets clustered (left side). For each of the targets, its closest neighbours are removed

placed in the open configuration at the location of the sampled pose, and if there are no collisions between the gripper and the target object or the fixed obstacles in the scene, then the pose is added to the targets set. The illustration of the verification process is presented in Fig. 6 .

The process of sampling and verification is repeated, until there are $N_{\text {tests }}$ elements in the targets database. At this point, the size of the samples database $N_{\text {samples }}$ will depend on the geometry of the target object, with $N_{\text {samples }} \geq N_{\text {targets. }}$. The samples and the targets sets after the verification step are illustrated in Fig. 5 b.

\subsubsection{Grasp Filtering}

The heuristic sampling is largely biased by the object geometry, some areas of the object are therefore more densely covered by grasps than others. The filtering provides an approximate grasp un-biasing such that the filtered grasp targets are close to uniformly distributed in $S E 3$ over the possible grasp space. This property is required in order to evaluate coverage which describes how large the success space (in $S E 3$ ) is in relation to the possible grasp space (in $S E 3$ ). Furthermore, it is desirable to reduce the size of the simulated grasp set due to computational reasons; otherwise they may contain tens of thousands of grasps. Thus, an intermediate step of filtering the grasp databases is introduced. We apply a greedy algorithm that iteratively selects the highest quality grasp $g_{\text {highest }}$ and removes any grasp that is within the weighted distance $D\left(g_{\text {highest }}, g_{i}\right)<1$ (introduced below) of the selected grasp. The illustration of the filtering step is presented in the Fig. 7.

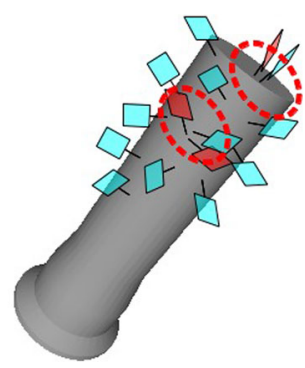

(removed targets are shown in red; the dashed ellipses illustrate the filtering distance)

A grasp pose is defined in $S E 3$ using Euclidean position and Equivalent Angle Axis $(E A A)^{2}$ for orientation:

$g_{i}=\left\{x_{i}, y_{i}, x_{i}, e a a_{x i}, e a a_{y i}, e a a_{z i}, e a a_{\theta i}\right\}^{T}$

The weighted distance metric is an infinity metric:

$D\left(g_{i}, g_{j}\right)=\left\|A \cdot\left(g_{j}-g_{i}\right)\right\|_{\infty}$

where $A=\operatorname{diag}\left(d^{-1}, d^{-1}, d^{-1}, \theta^{-1}, \theta^{-1}, \theta^{-1}, \theta^{-1}\right)$. In this work we selected the coverage filtering distance to be $d=0.001 \mathrm{~m}$ and $\theta=15^{\circ}$.

The algorithm above will not completely un-bias the grasp targets, but it will provide a decent approximation of well-behaved sets. At this point, the sizes of the samples and targets sets are reduced, and they contain $N_{\text {filteredsamples }}$ and $N_{\text {filteredtargets }}$ after filtering.

The distribution of grasps within targets and samples sets after the filtering step is also shown in a sample simulation scenario for a cylindrical object in Fig. 5c.

\subsection{Grasp Simulation}

The next step (Fig. 4c) is to evaluate all the filtered grasp targets and quantify their grasp quality. The evaluation is performed through dynamic simulation, in which the object is grasped according to each of the grasp targets in the filtered targets set generated in the grasp-planning phase.

For simulating the grasp execution, we use the RobWorkSim simulator, which is a part of the RobWork package [30]. The simulator uses the ODE physics

${ }^{2}$ represents an axis $(\mathrm{x}, \mathrm{y}, \mathrm{z})$ and an angle $\theta$ rotated around that axis. 
engine. The simulation allows for easy definition of the environment, including object models, kinematic and dynamic parameters of devices, as well as properties of the task context.

A grasp is considered to be successful, if the following conditions are met after the simulated grasp execution:

1. The object remains in the gripper with the GWS (Grasp Wrench Space [31]) (see Section 4.4) exceeding a specified lower limit $w_{\min }$.

2. No collisions with fixed obstacles in the scene occurred.

3. The interference (i.e. a measure of negative interaction of the gripper with the environment, explained below) at the end of the experiment does not exceed a specified interference limit $i_{\text {max }}$.

The interference is introduced as the measure of undesired gripper interaction with movable objects in the scene (e.g. other workpieces). Interference is calculated as a total sum of differences between the poses of all movable objects from before $\left(T_{i}^{\text {start }}\right)$ and after $\left(T_{i}^{\text {end }}\right)$ grasping:

$$
I=\sum_{i=0}^{\text {nobjects }}\left\|T_{i}^{\text {end }}-T_{i}^{\text {start }}\right\|
$$

Successful grasps are added to the successes set. If a grasp failed due to interference limit violation, then it is added to the interferences set. Otherwise, the grasp become part of the failures set. The distribution of the grasp simulation results is depicted in Fig. 5d.

A small fraction of simulations (usually around $\% 1$ ) becomes unstable due to limitations of the physical engine. The results of these simulations are discarded.

\subsection{Calculating Gripper Quality}

The gripper quality indices are computed after performing all grasp simulations, based on the statistical results of the simulations and the populations of the sets: successes, interferences and failures. The sizes of the results sets are denoted as $N_{\text {successes }}$, $N_{\text {interferences }}$ and $N_{\text {failures }}$, respectively.

We introduce the following gripper quality indices, which we explain in detail in Section 4: success index, robustness index, coverage index, coverage index, wrench index, alignment index, feasibility indices.

\subsection{Gripper Design Optimization}

Based on the obtained gripper evaluation, it is possible to either choose a good gripper design, or improve the existing design using optimization techniques. In Section 4.7 , we propose a gripper quality objective function, that combines the computed gripper quality indices into a single objective function that can be optimized using a numeric optimization method.

In our experiments (Sections 5.4 and 5.6), we optimize the objective function using Nelder-Mead downhill algorithm (also known as the downhill Simplex method) [1]. We chose the method based on its simplicity, and fairly robust performance in the presence of moderate noise.

\section{Quality Indices}

In this section, we introduce the proposed gripper quality indices that we use to determine the overall gripper quality for a specific grasping context. The indices are meant to be independent of the specific design parametrization.

The indices capture the most important aspects of gripper usage, including the success ratio, versatility of the gripper, robustness, alignment, and the feasibility of the design (Sections 4.1-4.6). We also propose a combined gripper quality objective function that is calculated as a weighted average of the indices (Section 4.7). The objective function is later used for optimization purposes.

\subsection{Success Index}

This is the most basic and intuitive index, capturing the success probability of a randomly planned grasp for the evaluated gripper design, given the grasp context constraints. The success index is calculated as a statistical measurement.

We define the success index as:

$S=\frac{N_{\text {successes }}}{N_{\text {filteredtargets }}}$

where $N_{\text {successes }}$ is the number of successfully executed grasps from the filtered targets database and 
$N_{\text {filteredtargets }}$ is the total number of grasps tried in the dynamic simulation. The value of the success index ranges naturally from 0.0 to 1.0 . It is a good test to give a preliminary assessment of the gripper design's performance.

\subsection{Robustness Index}

Uncertainties in grasping, pose detection and calibration have an impact on the performance of a robot cell. When a system has large uncertainties due to its sensing system, then it is required that the gripper is able to compensate for this uncertainty and still grasp reliably. The gripper robustness index evaluates how robust the performance of a specific gripper design is when adding uncertainties to the object location relative to gripper.

To assess the robustness of the gripper design, we introduce another iteration of simulations. In that simulation we take a set of grasps which are based on the successful grasps from the first round of simulation with additional noise introduced in form of perturbation that is drawn from a distribution modelling the pose estimation uncertainty.

The robustness index is calculated as follows:

$R=\frac{N_{\text {successes }}^{*}}{N_{\text {successes }}}$

where $N_{\text {successes }}^{*}$ is the number of successfully executed grasps in the perturbed grasps set, and $N_{\text {successes }}$ is the number of original successes.

The accuracy of the robustness measurement as described here is biased by the size of successes set $N_{\text {successes. }}$. When the success ratio of the evaluated gripper is particularly low, the robustness measurement is less reliable.

\subsection{Coverage Index}

There are grasping tasks, in which it is important that the gripper is able to perform grasps from many different directions. This might be for example bin picking, where many approach direction potentially are blocked when grasping. A few very high quality grasps might not be sufficient to compute grasps in highly cluttered scenes, simply because objects in the environment may collide with the gripper and thereby strongly limit the successful grasp space. We intend the coverage index to capture gripper agility in this type of conditions. In general, a gripper with a high coverage is very maneuverable within the task constraints, which may enable a higher success rate and faster execution.

The coverage evaluation is based on comparing the grasp success space of the actual gripper with the grasp success space of an abstract, infinitely thin and unbreakable gripper that does not generate any collisions. Such an ideal gripper, for the case of parallel-finger gripper, only requires two nearly parallel surfaces on opposite sides of the object to perform a successful grasp.

The coverage is computed as the ratio between the number of possible grasps of the specific gripper and the number of possible grasps of the ideal gripper. Due to the filtering step described in Section 3.1, we assume a correlation between the number of grasps and the grasp volume, and thus we make an identity $N_{\text {ideal }}=N_{\text {filteredsamples }}$. We infer that the coverage index defines the size of the grasp success space relative to the ideally possible success space. The complete success space is only dependent on the object and not the gripper, thereby enabling comparison across grippers.

The coverage index is calculated as:

$C=\frac{N_{\text {gripper }}}{N_{\text {ideal }}}=\frac{N_{\text {successes }}+N_{\text {interferences }}}{N_{\text {filteredsamples }}}$

where $N_{\text {gripper }}$ is the number of all grasp successes in the gripper evaluation plus the number of grasps that failed due to interference (since for coverage we only consider the object shape). The $N_{\text {filteredsamples }}$ represents the un-biased (filtered) grasp space of the ideal gripper.

\subsection{Wrench Index}

The wrench index provides more detailed information about the quality of grasps than the simple statistics of success ratio binary outcomes. The index is based on the average of grasp quality measurements of individual simulated grasps.

For the individual grasp quality evaluation, we use the Grasp Wrench Space (GWS) measure which was originally introduced in [31]. The particular flavour of GWS implementation varies in the literature [32]. In 
a

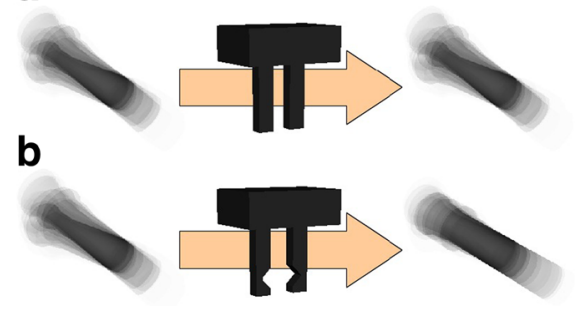

Fig. 8 Gripper alignment properties. a - A gripper with flat fingers does not align the cylindrical workpiece. $\mathbf{b}-$ A gripper with prismatic cutout (b) aligns the workpiece into a predictable stable pose leaving it only one degree of rotational freedom

our work, we use the definition of GWS from [33], and for the details on the implementation we refer to [34].

The GWS measurement of the grasp indicates the level of forces and moments that is required to dislodge the grasped objects from the gripper. It is also a useful metric for considering the possible levels of acceleration during any post-grasp object manipulation tasks. High GWS index implies a reduction of task execution time.

The wrench index of the gripper is defined so as to capture the overall average quality of all successfully executed grasps. We calculate the index as:

$W=\frac{1}{N_{\text {successes }}} \sum_{i=1}^{N_{\text {successes }}} w_{i}$,

where $w_{i}$ denotes the GWS measurement of $i$-th individual successful grasp.

\subsection{Alignment Index}

It is usually not enough that a gripper can execute a set of arbitrary grasps successfully. The gripper design is expected to compensate for uncertainties existing in the robotic setup due to, e.g. imprecise calibration, or pose estimation. Moreover, after the grasping, the robot is very often required to perform a task with the grasped object, which requires a great degree of control and precision (e.g. peg-in-hole action). Thus, it is desirable to evaluate the gripper performance in relation to its aligning abilities.

We define the alignment index of the gripper as its ability to place the grasped object in a predictable pose with reliability, while subject to uncertainties in the object pose before grasping. As an example, let us consider grasping of a cylindrical object with two cases of parallel jaw grippers: one with flat fingers (Fig. 8a), and one with a prismatic cutout (Fig. 8b). The gripper with a cutout is able to force the grasped object into a predictable pose (in rotation).

We derive the alignment capability of the gripper in the following way (see Fig. 9). Let us consider the sets of poses of the object in relation to the TCP of the gripper before (the set $\mathbf{P}$ ) and after grasping (the set $\mathbf{P}^{*}$ ). Each of the poses $p_{i} \in \mathbf{P}$ is mapped into a respective pose $p_{i}^{*} \in \mathbf{P}^{*}$ due to interaction between the gripper and the object during grasp execution.

Within the set $\mathbf{P}^{*}$ of poses after grasping, it is possible to find $0, \ldots, n$ stable pose models $S_{j}$ (it is possible to devise various definitions for a stable pose model - we propose a stable pose model below). Each of the models $S_{j}$ in the set $\mathbf{P}^{*}$ will possess a basin of attraction in the set $\mathbf{P}$. We measure the generalized radius $r_{j}$ of the $j$-th basin of attraction as the variance of distances $\left\|p_{i}-S_{j}^{*}\right\|$ of poses from before the grasping, to the stable pose model $S_{j}$ they belong to after grasping. We calculate the alignment index as a weighted average of generalized radii $r_{j}$ with weights
Fig. 9 The set of object poses with relation to gripper TCP before grasping $\mathbf{P}$ is mapped into the set $\mathbf{P}^{*}$ after grasping. Stable pose model $S_{j}$ is found in the set $\mathbf{P}^{*}$ with a corresponding basin of attraction with a generalized radius $r_{j}$ in set $\mathbf{P}$

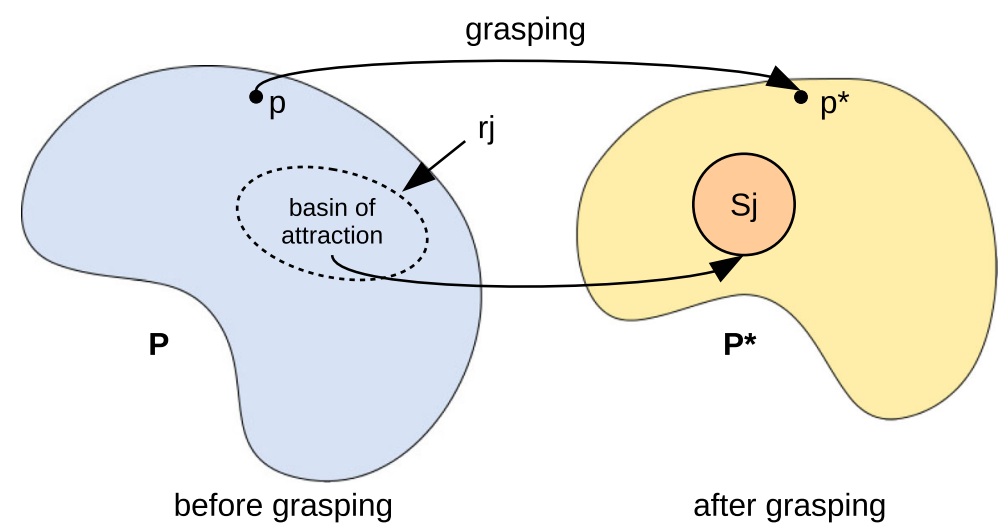

after grasping 
based on the number of poses belonging to each stable pose model $S_{j}$ :

$A=\frac{1}{N_{\text {grasps }}} \sum_{j=0}^{k} n_{i} r_{j}$

where $N_{\text {grasps }}$ is a number of grasps which ended with either success or interference, $n_{i}$ is the number of grasps belonging to the $j$-th pose model, and the $r_{j}$ is the generalized radius of $j$-th stable pose basin of attraction.

Stable Pose Model The desired pose, into which the gripper is expected to align grasped objects, can either be supplied as a parameter by user, or it can be found by employing pose model extraction from the data. The pose definition is particularly difficult in the case of symmetrical objects, for which an acceptable pose is defined by a region rather than a point in SO3. We employ a notion of a stable pose (defined in [35]). We repeat the definition here for the sake of completeness.

A stable pose $S$ is defined as a connected set of poses in $S O 3$, representing poses that are allowed for a physical object placed on a flat supporting surface. The object has therefore 1 to 2 rotational degrees of freedom, and when plotted, the unit vectors $\hat{x}, \hat{y}$ and $\hat{z}$ belong to either parallel planes or bands on an unit sphere. At least one such plane is always defined. A stable pose can be described using a normal vector shared by the planes, and the individual plane distances:

$S=\left\{\mathbf{n}, d_{1}, d_{2}, d_{3}\right\}$

When the desired stable pose is not provided, we use RANSAC to extract stable poses of the objects from the simulation data before and after grasping. We then calculate the alignment index, using these poses in (Eq. 9).

\subsection{Design Feasibility}

For designing gripper fingers, it is not only important that they score highly on the quality indices introduced so far, but also that the design is physically feasible. This means that a good gripper jaw design should be robust enough to handle the stress of grasping operation without breaking or degradation. It is also good to have a gripper design that is easy and cheap to manufacture. This is especially important in the case of parallel-finger grippers, in which the fingers are commonly obtained by using 3D printing. In this case, reducing the printed volume, while still maintaining structural strength, allows for shortening of production time of the fingers.

The design feasibility concept is not universal to all types of grippers, and it has to be defined by the system user as to adapt it to those types accordingly. It can also encompass multiple independent design qualities, and can therefore comprise of several individual indices. We will denote these as feasibility indices $F_{j}$.

For our case of parallel finger grippers, we propose a design feasibility metric that takes into account the general finger volume, and the projected maximum stress in form of two separate indices: volume index $F_{1}=V$, and stress index $F_{2}=\zeta$.

Volume Index We calculate the volume index $V$ based on the ratio of the volume of finger geometry $V_{\text {finger }}$ to the predefined volume limit $V_{\max }$ :

$F_{1}=V=\left\{\begin{array}{lll}1-\frac{V_{\text {finger }}}{V_{\max }} & \text { when } & \frac{V_{\text {finger }}}{V_{\max }} \leq 1 \\ 0 & \text { when } & \frac{V_{\text {finger }}}{V_{\max }}>1\end{array}\right.$

Stress Index For computing the stress index $\zeta$, the worst case scenario is considered - the grasping force acting at the very tip of the jaw, perpendicular to its axis, where it produces the highest bending moment. This placement is reasonable, because due to uncertainties in the grasping process, such an unfavourable condition may occur - possibly due to collision or interference with other workpieces. The bending moment is calculated for selected points along the length of the finger:

$M=F_{\text {grasp }} \cdot x$

where $F_{\text {grasp }}$ is the grasping force provided by the actuation mechanism, and $x$ is the distance along the gripper axis from the force placement location at the tip of the finger.

The stress is then computed for crossections of the finger's geometry:

$\sigma=\frac{M}{I_{\text {crossection }}}=\frac{6 M}{b h^{2}}$

where $I_{\text {crossection }}$ is the second moment of area of the crossection, $b$ is the breadth of the crossection, and $h$ is the height of the crossection. The process of finding the maximum stress value for the finger geometry is presented in Fig. 10. 
$\mathrm{h}$

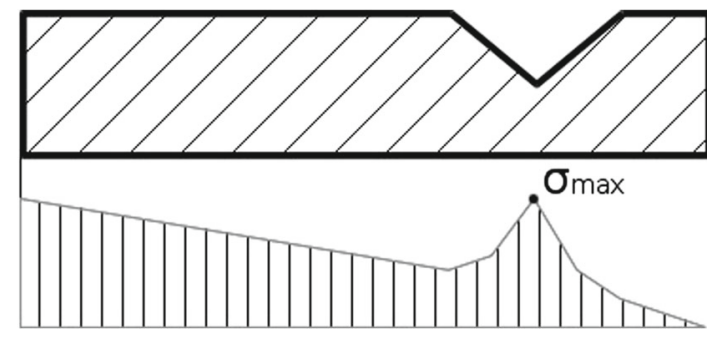

Fig. 10 The illustration of finding the maximum stress value for worst-case placement of the grasp force (tip of the finger ois $\mathrm{n}$ the right side). The maximum stress $\sigma_{\max }$ is at the peak located at the center of the finger cutout

The stress index $\zeta$ is finally defined based on the the ratio of $\sigma_{\max }$, i.e., the highest value of stress found for the given jaw's geometry to the expected stress level defined by the user $\sigma_{\text {limit }}$ :

$F_{2}=\zeta=\left\{\begin{array}{lll}1-\frac{\sigma_{\max }}{\sigma_{\text {limit }}} & \text { when } & \frac{\sigma_{\max }}{\sigma_{\operatorname{limit}}} \leq 1 \\ 0 & \text { when } & \frac{\sigma_{\max }}{\sigma_{\text {limit }}}>1\end{array}\right.$

\subsection{Gripper Quality Objective Function}

Finally, we provide a gripper quality objective function which combines the aforementioned indices into a single value. This provides a quantitative overall assessment of the gripper evaluation, and can be used to distinguish different gripper designs based on their merit. The objective function is useful for optimization purposes, where we seek to obtain a maximum quality of a design. Different users might seek optimization of their gripper designs for different purposes. This can be controlled by supplying custom weights for respective indices.

We propose using a weighted product metric to combine selected gripper quality indices into a single quality objective:

$Q=\left(\prod_{i=1}^{N} q_{i}^{w_{i}}\right)^{1 / \sum_{i=1}^{N} w_{i}}$

where $\mathbf{q}=\left[S, R, C, W, F_{1}, F_{2}, \ldots\right]$ is the vector of $N$ gripper quality indices, including $S, R, C, W$ and $F_{j}$ (respectively: success, robustness, coverage, wrench, feasibility indices), and the $w_{1}, w_{2}, \ldots$ are the respective weights.

The objective function we use in subsequent optimization experiments combines crucial quality indices of the gripper. This, we argue, provides a flexible evaluation of the gripper.

\section{Experiments}

In this section, we describe the experiments we performed to verify our proposed approach. We start with a detailed description of our setup (Section 5.1), in which we present the scenes and task that we used for our experiments, as well as a simple gripper parametrization.

In Section 5.2 we show that the quality indices introduced in Section 4 are able to distinguish between different designs of grippers generated by the proposed parametrization. Next in Section 5.3, we present and discuss the characteristics of the objective function introduced in Section 4.7 in the domain of the presented parametrization.

Based on the discussion of objective function properties, we show that it is possible to decouple certain pairs of gripper parameters and quality indices, and thereby reduce the dimensionality of the optimization problem. As an example, we consider two cases: (1) the influence of cutout parameters on the alignment index in the rotorcap side grasping scenario (Section 5.4), and (2) the influence of selected gripper design parameters on coverage index for the pump housing object grasping scenario (Section 5.6). In both of these cases we discuss the obtained results in light of the previous studies and our engineering experience. In addition, in the case of rotorcap gripper optimization for alignment improvement, we have performed a set of grasping experiments both in simulation and using real 3D printed fingers to prove the validity of obtained results. This experiment is described in Section 5.5.

\subsection{Experimental Setup}

In this section we will present the scenes and tasks that we subsequently use in our simulated experiments. We will also introduce a simple gripper parametrization using 12 bounded numerical parameters.

\subsubsection{Scenes, Objects and Tasks}

Our experiments involve the simulation of several basic grasping tasks of two objects, the rotorcap and the pump housing object (see Fig. 11). Both objects originate from real world industrial automation problems and they are meant to represent a sample of typical grasped workpieces. We decided to choose 
Fig. 11 Objects grasped in our experiments: rotorcap (on the left), pump housing object (on the right)
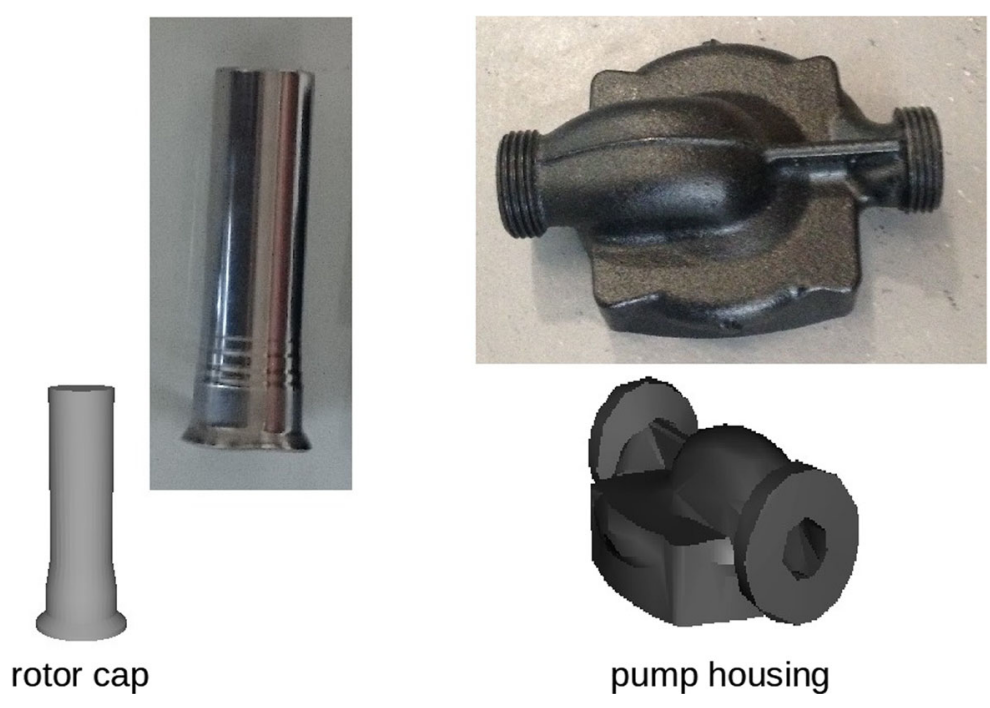

one object with a simple geometry, in order to have an experimental setup in which we can qualitatively verify our method by assessing the results with engineering knowledge and intuition, and a second object with more elaborate shape. The objects are defined to have a mass of $0.1 \mathrm{~kg}$, and the material is defined to have the physical properties of aluminum in the context of simulation.

In our experiments, we will consider two scenes which include the objects introduced above. For each of the scenes, we also introduce three simple grasping tasks, based on the direction of approach. The scenes and tasks are illustrated in Fig. 12. For both the rotorcap, and for the pump housing, we consider an arrangement of objects on a flat surface resembling a belt. The rotorcap scene is meant to serve for the purpose of illustrating the qualitative performance of our evaluation method, while the more complicated pump housing scene will be used to demonstrate the versatility of our approach.

In each scenario the base task is a simple grasping action: the gripper approaches the object, the grasp is executed, then the object is lifted vertically. We vary the three defined tasks by restricting the approach direction: first we allow grasping from every direction (Fig. 12(1)), then we constrain the gripper approach to top grasps (Fig. 12(2)), and for the third we only execute grasps from the side (Fig. 12(3)). When constraining the approach direction to be either vertical or horizontal, we allow for some deviations in the direction to represent the uncertainty in the grasping system. Angular differences in the range of $45^{\circ}$ are allowed.

The design feasibility indices introduced in Section 4.6 require custom limits imposed on the maximum stress in finger, and the volume of material used.
Fig. 12 Scenes used for gripper evaluation experiments. The top row shows the rotorcap scene and the bottom row shows the pump housing scene. Three grasping tasks are presented: (1) grasping from all directions, (2) direction of approach restricted to vertical (from the top), (3) approach direction restricted to horizontal (from the side)

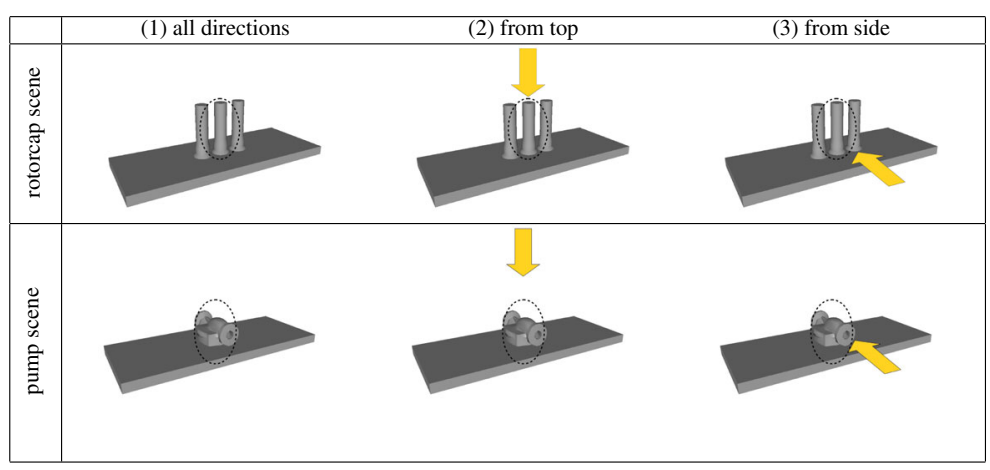


In our experiments, we picked the values of $V_{\max }=$ $100\left[\mathrm{~cm}^{3}\right]$, and $\sigma_{\text {limit }}=100[\mathrm{kPa}]$.

\subsubsection{Gripper Parametrization}

In our experiments, we use a gripper parametrization of moderate complexity, which allows us to verify that our method yields correct results, and yet includes gripper features (such as chamfering, and cutout) commonly encountered in industrial setups. A simple parametrization allows us to reduce the dimensionality of the search space, to focus more on showing the general feasibility of our optimization approach. It should be noted, that more complex parameterizations are possible, and our method would be easily extendable to allow for various other features in the jaw geometry such as finger tapering, asymmetry, or tabs preventing jams. The parametrization used in our experimental setup is presented in Table 1 and is further illustrated in the Fig. 13.

\subsection{Hand-Picked Gripper Designs Evaluation}

In this section, we will provide and discuss results of the quality evaluation of a number of selected gripper finger geometries.
Table 1 The parametrization of a gripper design

\begin{tabular}{|c|c|c|c|}
\hline $\mathrm{N}$ & Name & Range & Notes \\
\hline \multicolumn{4}{|c|}{ General dimensions } \\
\hline 1 & length & $0-0.2 \mathrm{~m}$ & length of a finger \\
\hline 2 & width & $0-0.025 \mathrm{~m}$ & measure of finger's footprint \\
\hline 3 & depth & $0-0.025 \mathrm{~m}$ & $\begin{array}{l}\text { breadth of the grasping surface of } \\
\text { the gripper }\end{array}$ \\
\hline \multicolumn{4}{|c|}{ Chamfering parameters } \\
\hline 4 & chamfer depth & $0-1$ & $\begin{array}{l}\text { expressed as a ratio to the finger's } \\
\text { width }\end{array}$ \\
\hline 5 & chamfer angle & $0^{\circ}-90^{\circ}$ & $\begin{array}{l}\text { angle of the chamfering; higher } \\
\text { value reduces the gripper's foot- } \\
\text { print considerably }\end{array}$ \\
\hline
\end{tabular}

$0-0.2 \mathrm{~m}$

6

TCP offset

position of the TCP of the gripper in relation to its base; this is also the position of the cutout; this should be less than the gripper's length

7

cut depth

$0-0.025 \mathrm{~m}$

8

cut angle

$0^{\circ}-90^{\circ}$

9

cut tilt

$-90^{\circ}-90^{\circ}$

Miscellaneous parameters

10

jawdist

$0-0.05 \mathrm{~m}$

11

stroke

$0-0.05 \mathrm{~m}$

12

force

$0-50 \mathrm{~N}$ depth of the cutout; this should also be lower than the finger's width

angle between the walls of the prismatic cutout

angle of the cutout respective to normal to the gripper top surface

the distance between the gripper's fingers when in the 'closed' configuration

the range of movement of the gripper's fingers; this must be lesser or equal to the opening value

the force the actuation mechanism can provide for grasping 
Fig. 13 The parametrization of the parallel gripper design
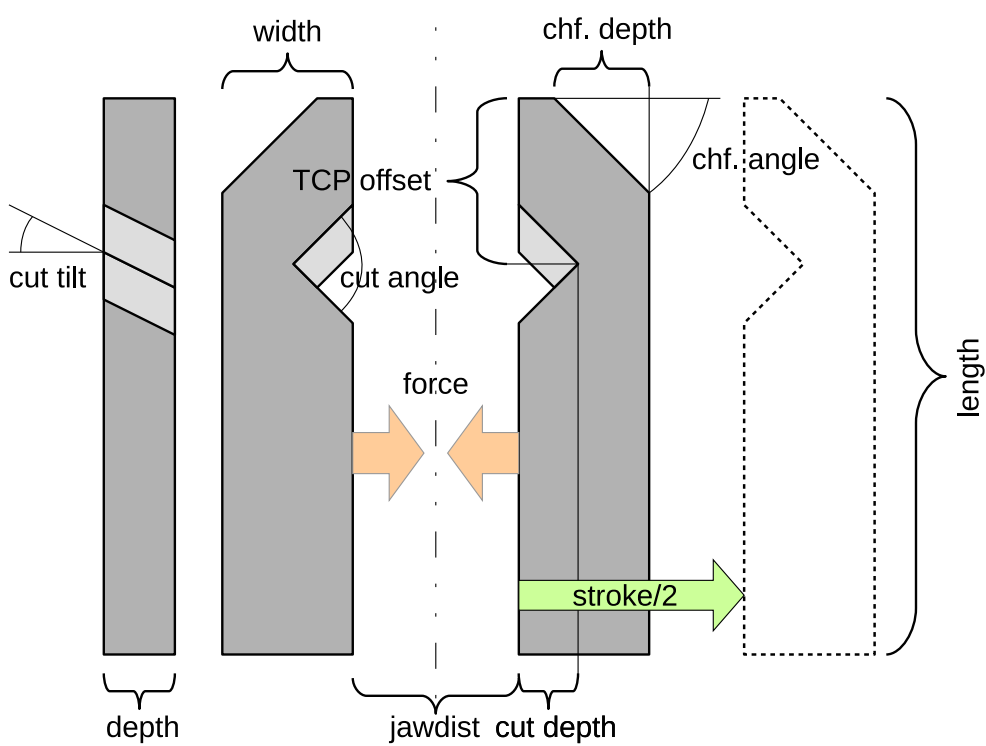

For this experiment, we have created one degree of freedom grippers with six fingers of different geometry based on features commonly encountered in real design (see Fig. 14a-f): a - a standard box-shaped finger, $\mathbf{b}-\mathbf{a}$ chamfered finger tapered towards the endpoint, $\mathbf{c}-\mathbf{a}$ finger with prismatic cutout, $\mathbf{d}-\mathbf{a}$ finger with both chamfering and cutout, $\mathbf{e}-$ a thin finger, and $\mathbf{f}-\mathrm{a}$ fat box-shaped finger. The grippers equipped with these fingers have been tested on the rotorcap scene, using all three tasks (see: Section 5.1.1). Each of these 18 evaluations ( 6 grippers applied to 3 tasks) is based on $n=1000$ simulated grasps.

The evaluation results and the finger parameters are presented in the Fig. 14 and can be interpreted as follows: for a given gripper design, in all scenarios the feasibility indices (i.e., stress index $\zeta$ and volume index $V$ ) remain constant, due to being only dependent on the finger geometry. It is immediately obvious that the presence of cutouts or chamfering weakens the structural integrity of the gripper, while at the same time it reduces the volume of material needed (designs c \& d). The particular value of these indices depends on selected limits for finger strength and volume (see Section 4.6), and will therefore change according to custom values provided by the user. Fingers of higher bulk, and more resistant material are possible. Not surprisingly, the gripper designs $\mathbf{e}$ and $\mathbf{f}$ exhibit the upper and lower extremes of the volume and stress indices ( $V$ and $\zeta$ respectively).
It can be seen that the presence of finger chamfering offers advantages in terms of success ratio $S$ (see Section 4.1) both for picking from all directions, and from the side (see designs $\mathbf{b}$ and $\mathbf{d}$ ). This is explained by the gripper now exhibiting a smaller footprint which reduces interference. While grasping from the top, the environment appears less cluttered, and in that case the influence of chamfering is negligible. The same advantage is conferred by reducing general finger dimensions, as can be seen in gripper $\mathbf{e}$. This is however less desirable due to the lower values of the stress index $\zeta$ (see Section 4.6).

Both wrench $W$ and alignment $A$ indices (see Sections 4.4 and 4.5) are improved when a cutout is introduced in the rotorcap grasping from the side scenario (designs $\mathbf{c} \& \mathbf{d}$ ). A change in these indices is not pronounced for other directions of grasping, which is due to the cutout being oriented perpendicularly to the finger axis, making it less likely to be helpful in these scenarios. Wrench $W$ and alignment $A$ also increase with higher finger depth, as can be seen in gripper $\mathbf{f}$, but at the cost of volume index $V$.

The robustness index $R$ (see Section 4.2) tends to decline for gripper designs with a cutout feature (c and d). This is because grasps in which the object is misaligned with the cutout are slightly more likely to fail.

The coverage index $C$ (see Section 4.3) tends to have a higher value for grippers with chamfering, and 


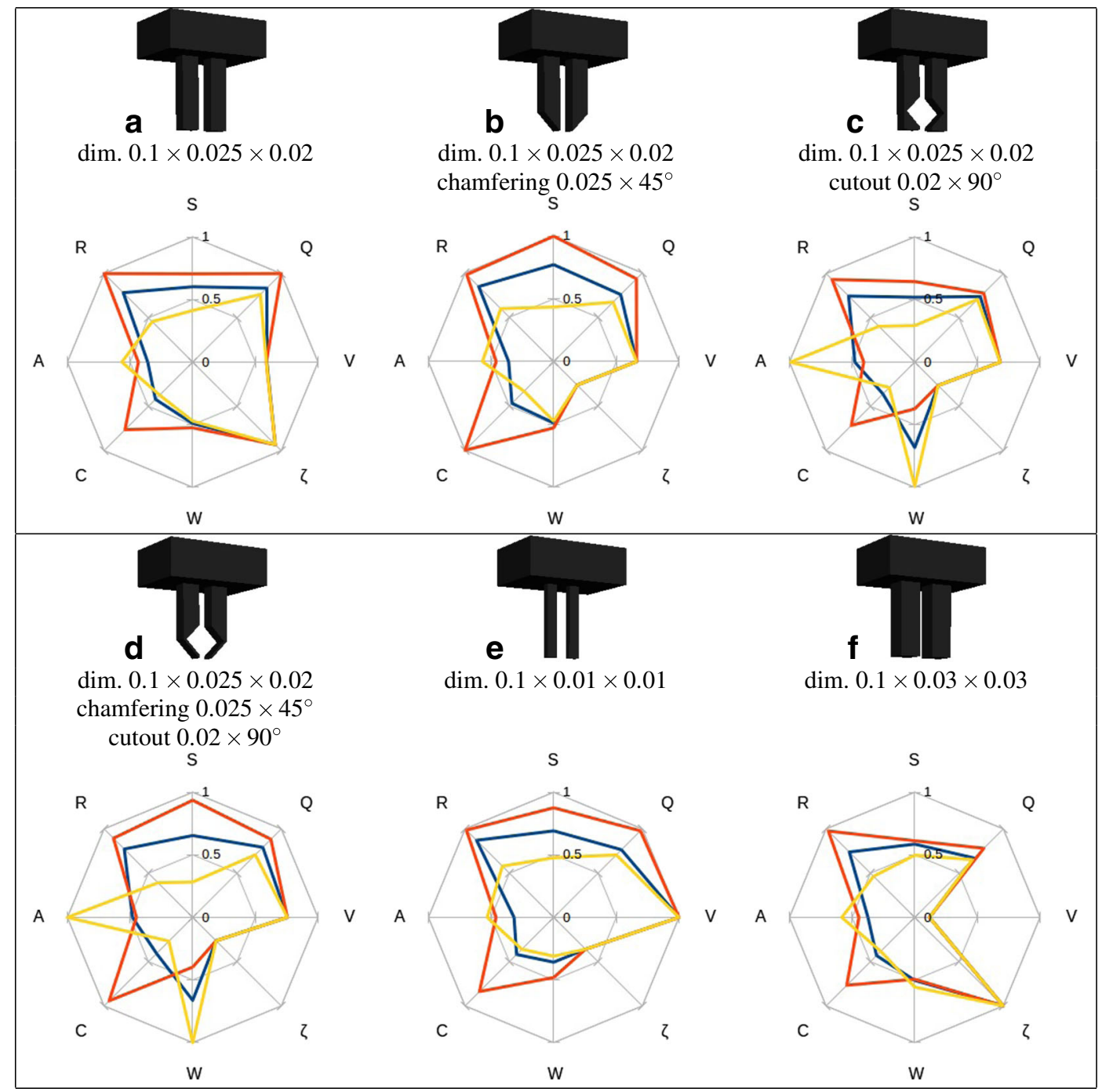

Fig. 14 Quality evaluations of hand-picked gripper designs: a standard, b with chamfering, $\mathbf{c}$ with prismatic cutout, $\mathbf{d}$ with chamfering and cutout, e thin, f fat. Each radar plot shows the gripper quality in three contexts for the rotorcap scene: all directions - color red, grasping from top - color blue, and grasping from side - color yellow. Each evaluation was performed for $n=1000$ grasps. Plots show relative index values between grippers, with 1.0 indicating the best score smaller footprint. The difference is not large, due to the rotorcap being a relatively featureless object.

The gripper quality has been calculated using Eq. 15, with weights $w_{i}$ all set to 1.0 to not put emphasis on any particular quality index.

\subsection{Quality Objective Function Properties}

We have been interested to see whether the influence of gripper design parameters as introduced in Section 5.1.2 can be intuitively understood. For that, we performed a set of experiments, in which we have picked a simple gripper design with flat fingers (see Fig. 14a, and varied a selected parameter to observe slices of the quality objective landscape. This is also important to assess whether the quality objective function is suitable for optimization purposes.

In Fig. 15a, we have considered the influence of the gripper length on the quality indices of the gripper in the rotorcap picking from the top scenario. The coverage index $C$ rises linearly, which is explained by the rotorcap height, as illustrated in Fig. 16a. A 

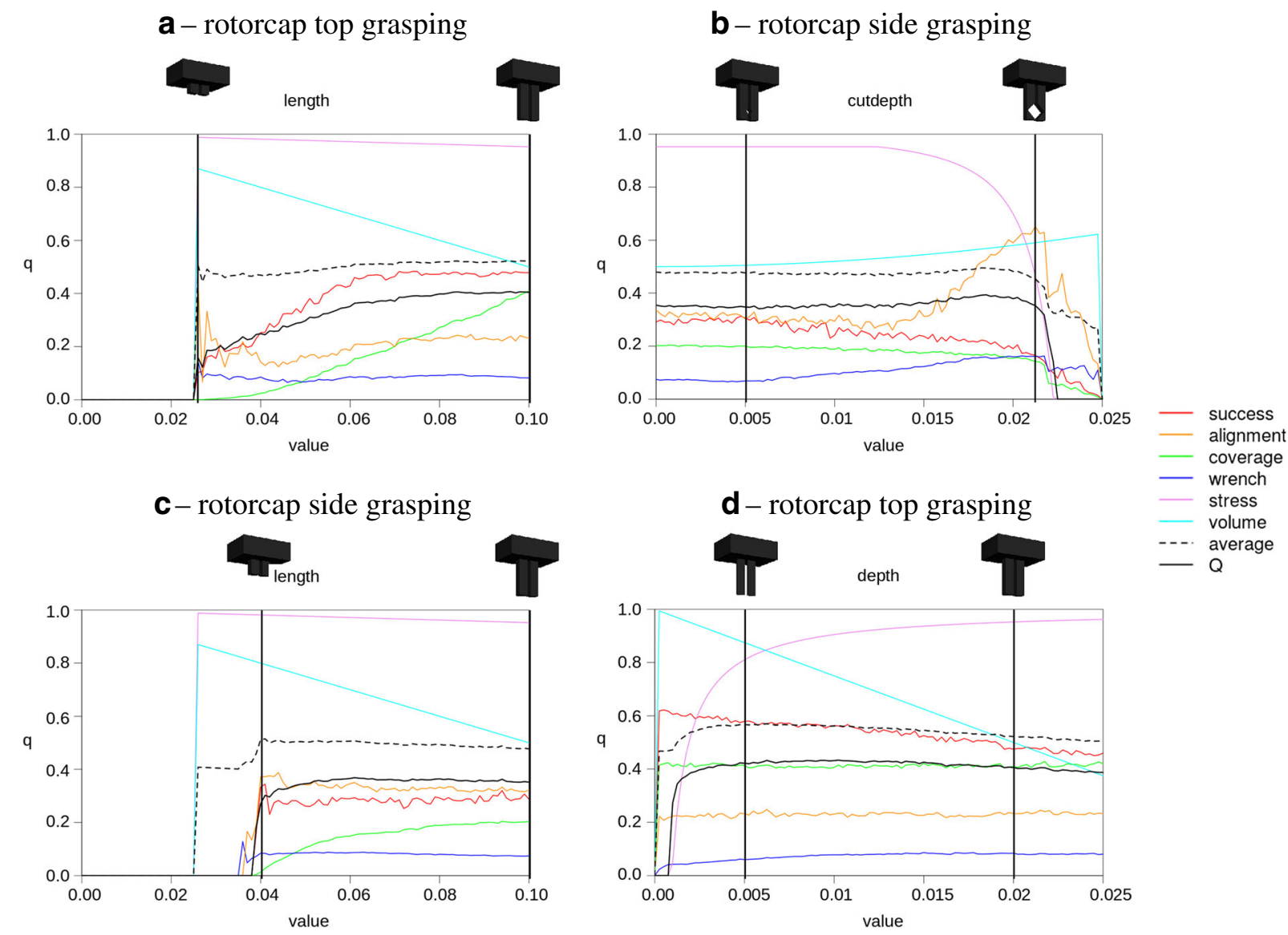

Fig. 15 Quality objective function plotted as slices of a complete space for a change in a single parameter in a template gripper design: $\mathbf{a}-\mathbf{Q}$ (length) for the rotorcap picking from the top scenario, $\mathbf{b}-\mathbf{Q}$ (cutdepth) for the rotorcap picking from the

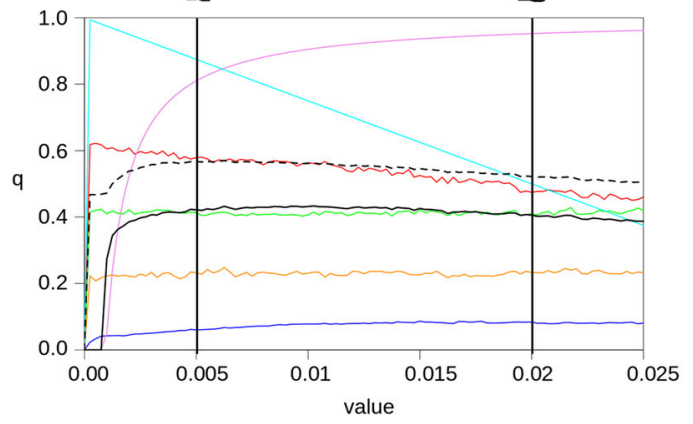

side scenario, $\mathbf{c}-\mathbf{Q}$ (length) for the rotorcap picking from the side scenario, $\mathbf{d}-\mathbf{Q}$ (depth) for the rotorcap picking from the top scenario. Quality evaluation based on $n=500$ grasps

longer gripper, when grasping from the top, is able to is explained by the the fact that for these only grasps reach even the bottom of the rotorcap. The peak for the alignment index $A$ for a very short finger length within a very small area at the top of the rotorcap are possible, as well as the fact that the base of the gripper

Fig. 16 a-Grasp coverage for a short, and for a long gripper in rotorcap picking from the top scenario. Long gripper is able to perform grasps on the side of the cylindrical object, while grasps of a short gripper are clustered on the top. $\mathbf{b}$ - In grippers with short fingers, the base influences the grasp result by helping to align the grasped rotorcap
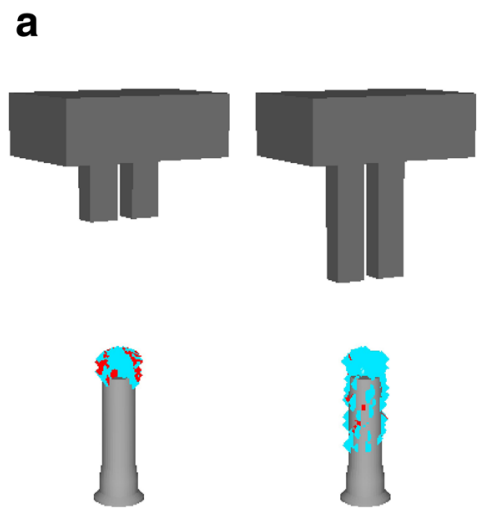

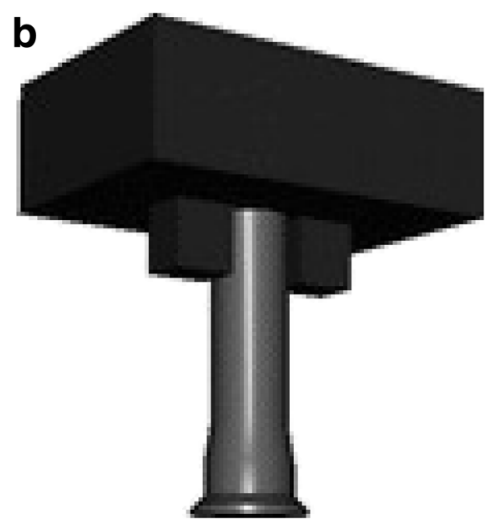


effectively serves as a 'third finger', stabilizing the piece even further (see Fig. 16b). The success ratio $S$ rises before hitting a plateau at finger length of $0.07 \mathrm{~m}$. The wrench index $W$ remains relatively constant. The dashed and solid black lines in the plot represent the average values of indices and the value calculated by using Eq. 15. The combined objective value obtained by using weighted product better reflects the overall quality of the gripper, and is well suited for optimization purposes.

Figure $15 \mathrm{~b}$ presents the gripper quality indices plotted for a change in the cut depth parameter for the rotorcap side grasping scenario (see Fig. 12). The cut depth is set to vary in range of $0 \mathrm{~mm}$ to $25 \mathrm{~mm}$, which is the full width of finger. The parameter that draws the most attention is the alignment index $A$, which increases prominently after a certain cut depth is achieved, and reaches its peak at the cut depth of $22 \mathrm{~mm}$. This depth corresponds to a depth that is best suited to embrace the thinner cylindrical part of the rotorcap object given the cut angle parameter set to $90^{\circ}$. A smaller secondary peak in alignment is noticeable for a bigger cut depth of $23 \mathrm{~mm}$, which is associated with the diameter of a thicker bottom part of the rotorcap. A drop in the success ratio $S$ at this value of the parameter is due to the cutout being now too deep to grasp the top part successfully. It is interesting to see that although a bigger cutout helps to improve the wrench index $W$ of the gripper, the alignment $A$ is only increased after a certain depth of the cutout is reached. Considerations of these rather complex dependencies are difficult to expect from an engineer performing manual finger design processes.

The influence of the gripper finger length on the quality indices for the rotorcap picking from the side scenario is presented in Fig. 15c. A qualitative difference of the plots for the same scene, but for a different approach direction is noteworthy (compare with Fig. 15a for the picking from the top scenario). The minimum length of the finger which yields success is increased in this task, which is explained by the fact that the gripper has to compensate for the belt offset when approaching from the side. Although the coverage $C$ increases with bigger finger length, the relation is no longer linear, because longer fingers do not help to reach more of the object geometry anymore. No significant alignment peak occurs, because the minimal finger length for which succesful grasps are possible no longer places the piece close to the gripper base, and hence this base cannot be exploited as a third finger as in the rotorcap top grasping scenario (Fig. 15c).

Finally, in Fig. 15d, we present the influence of the gripper finger depth on the quality objectives. This experiment has been executed in the rotorcap picking from the top scenario. In that task, we do not expect the finger depth to improve the surface contact area, and so the wrench index $W$ remains relatively constant with increased finger size. The success ratio $S$ drops with increased finger depth due to a higher chance of interference in the cluttered environment. Other indices seem to be relatively constant except the stress and volume indices ( $\zeta$ and $V$ respectively) that reflect the finger geometry changes.

Based on these evaluations, we argue that it is possible for each of the gripper quality indices in a given scenario to indicate a set of design parameters that influences that index. Thus, it is possible to decouple selected quality indices, and reduce the dimensionality of optimization problem by only considering a change in specific gripper design dimensions when trying to find optimal values for a single quality objective.

We will utilize this observation in the following two sections, by first showing the optimization of the alignment index $A$ by finding the best values of the cutout parameters in Section 5.4, and then the optimization of the coverage index $C$ using selected parameters in the pump housing object picking scene in Section 5.6.

\subsection{Gripper Alignment Optimization}

In this subsection, we perform optimization of the alignment index $A$ in the space of the cutout parameters (5-cut depth, 6-cut angle, and 7-cut tilt in Table 1) on the rotorcap scene for the picking from the side task (see Fig. 12). As argued in Section 5.3, we select these parameters as the most influential on the gripper alignment index. Prior to optimization, we have completed a low resolution exploration of the cutout parameters space, the results of which is presented in the form of 2-dimensional slice in Fig. 17. From that, we can conclude that there is a singular parameter configuration, which corresponds to the optimal gripper design in the context of the task.

Due to the level of noise still evident in the alignment evaluations despite increasing the number of 
Fig. 17 Alignment index of a template gripper in a rotorcap side picking scenario as a function of cut angle and cut tilt for selecte value of cut depth $=$ $22.5 \mathrm{~mm}$. Quality evaluation based on $n=500$ grasps

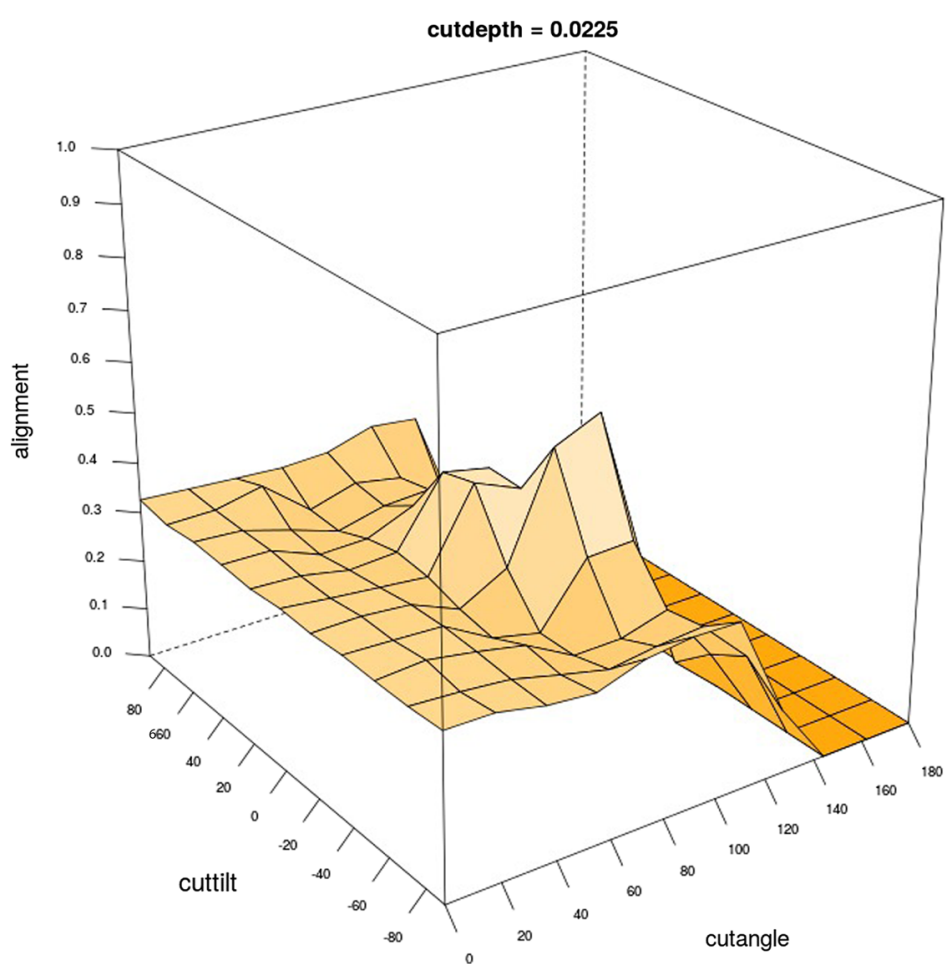

grasps per evaluation to $n=500$ and the presence of flat areas in the function plot, we have decided to define the objective function for this optimization experiment to include also the wrench index $W$, by selecting the following weights:

$$
\begin{array}{rll}
w_{S}=0 & w_{R}=0 \quad w_{A}=1 \quad w_{C}=0 \\
w_{W}=1 & w_{\zeta}=0.01 \quad w_{V}=0.01
\end{array}
$$

The wrench index $W$ is correlated with the alignment index $A$, and being less noisy and more sensitive to the cutout parameter changes, it helps to escape the initial stationary area in the optimization. Additionally, volume $V$ and stress $\zeta$ design feasibility indices were given a low weight of 0.01 . This is to ascertain that the gripper design obtained from the optimization procedure will be physically realistic.

A number of grippers $G=100$ were heuristically generated within the cutout parameter space as defined by ranges presented in Table 1 . The designs are all based on the standard gripper design (see Fig. 14a), with only cut depth, cut angle, and cut tilt parameters changing. The heuristics for the gripper generation were based on the sampled design evaluation - only grippers with $S>0, \zeta>0$, and
$V>0$ were approved. We have selected three of these grippers for further optimization. The gripper designs before and after the procedure are presented in Fig. 18.

The results show that despite variance in the initial parameter values, the optimization converges to similar designs which are best suited for the scenario. Even initial misalignment of the cutouts relative to the grasping direction are rectified by the procedure.

The cutout shape obtained from the optimization procedure seems suitable for cylindrical object picking when approaching the object in horizontal plane. The cutout tilt is perpendicular to the gripper fingers. The cutout dimensions fit the outer diameter of the rotorcap, so that the object is securely grasped within the gripper, with additional space to account for grasping uncertainties. The cutout is also not too large, to avoid the object falling out. As such the calculated shape seems to fulfill the objectives indicated in the qualitative guidelines, and overall appears to be a solid design according to our engineering expertise.

We have furthermore performed simulated experiments with structured perturbation emulating the uncertainty in grasping of the rotorcap object. For this, we have selected one of the optimal grippers designed using our method (rotor3_10) and a similar gripper 


\begin{tabular}{|c|c|c|c|}
\hline & rotor3_186 & rotor3_10 & rotor3_63 \\
\hline لِّ & $\begin{array}{l}\text { cut depth: } 0.0169 \\
\text { cut angle: } \\
163.75^{\circ} \\
\text { cut tilt: } 59.65^{\circ} \\
\text { A: } 0.069 \\
\text { Q: } 0.045\end{array}$ & $\begin{array}{l}\text { cut depth: } 0.0015 \\
\text { cut angle: } \\
114.15^{\circ} \\
\text { cut tilt: } 39.43^{\circ} \\
\text { A: } 0.313 \\
\text { Q: } 0.152\end{array}$ & $\begin{array}{l}\text { cut depth: } 0.0205 \\
\text { cut angle: } 98.41^{\circ} \\
\text { cut tilt: }-25.09^{\circ} \\
\text { A: } 0.304 \\
\text { Q: } 0.170\end{array}$ \\
\hline 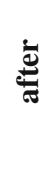 & $\begin{array}{l}\text { cut depth: } 0.0198 \\
\text { cut angle: } 99.96^{\circ} \\
\text { cut tilt: } 5.69^{\circ} \\
\text { A: } 0.683 \\
\text { Q: } 0.341\end{array}$ & $\begin{array}{l}\text { cut depth: } 0.0196 \\
\text { cut angle: } 94.09^{\circ} \\
\text { cut tilt: } 0.12^{\circ} \\
\text { A: } 0.635 \\
\text { Q: } 0.324\end{array}$ & $\begin{array}{l}\text { cut depth: } 0.0199 \\
\text { cut angle: } 94.99^{\circ} \\
\text { cut tilt: }-2.69^{\circ} \\
\text { A: } 0.645 \\
\text { Q: } 0.328\end{array}$ \\
\hline
\end{tabular}

Fig. 18 Gripper alignment optimization for the rotorcap scene and picking from the side task. Presented are the initial seed gripper designs (on the top) and the corresponding optimized designs (on the bottom)

featuring fingers with a molded geometric inverse of the rotorcap shape (a method used in e.g. [28]). The results of these simulations are presented in Fig. 19. The figure shows the grasp outcomes for grasps offset from the nominal grasp in $\mathbb{R}^{3}$, detailing the success space of the gripper. We have allowed for a position uncertainty of $\pm 2.5 \mathrm{~cm}$.

The rotor $3 \_10$ gripper was able to perform successfully for $36.36 \%$ of the perturbed grasps, while the molded gripper only had a success ratio of $26.90 \%$. These results clearly show the superiority of the optimized design.

\subsection{Comparison of Performance in Simulation and in a Real-World Setting}

After generating the finger geometry optimized for the alignment of the rotorcap object, as done in Section 5.4, we performed a set of experiments to compare the quality of the in simulation optimized gripper fingers with the quality achieved in a realworld setting. We have tested the grasping of the rotorcap with increasing offsets of the gripper TCP frame along the $x$ and $z$ translational axes and the rotations $R z$ (roll) and $R x$ (yaw) around the corresponding axes. In these experiments, we have executed the grasping and lifting action as described in Section 5.1.1.

The experimental setup with the offset axes is shown in Fig. 20. In the experiment, we use an UR5 robot equipped with the electrically actuated PG70 gripper with the fingers featuring the optimized rotor $3 \_10$ cutout (see Fig. 18). The fingers were printed with the length reduced, to accommodate the requirements imposed on the torque at the mounting points. The cutout geometry remains the same.

The following parameters were used to relate the simulation to the real-world task. We measured the
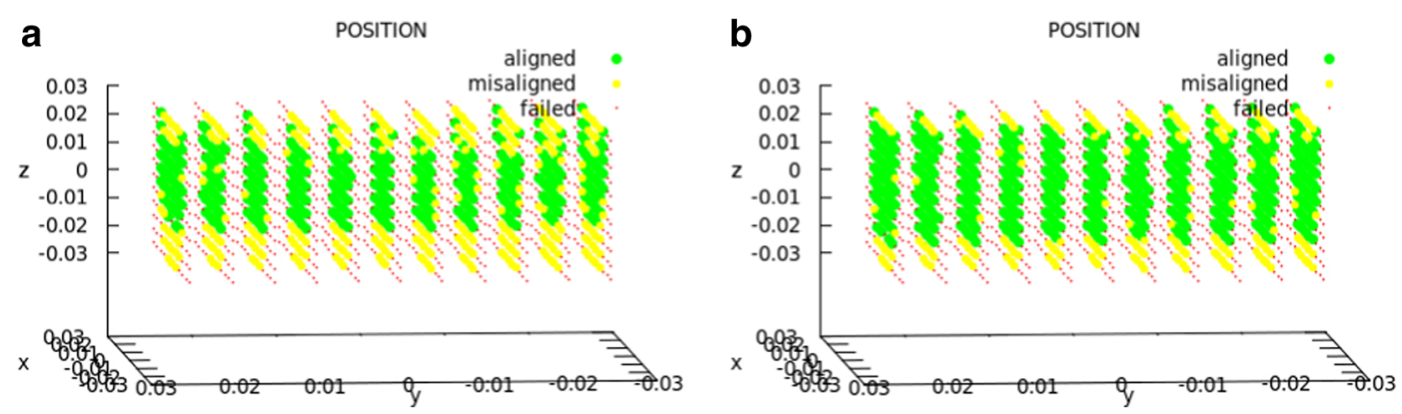

Fig. 19 Grasp success in the space of position uncertainty for a hand-designed gripper with a molded cutout (a), and for the optimized gripper rotor3_10 (b). The green color indicates complete grasp success, yellow - object misalignment, and red - grasp failure 
Fig. 20 The experimental setup to compare the grasp outcomes in simulation (on the left) and in the real world (on the right)

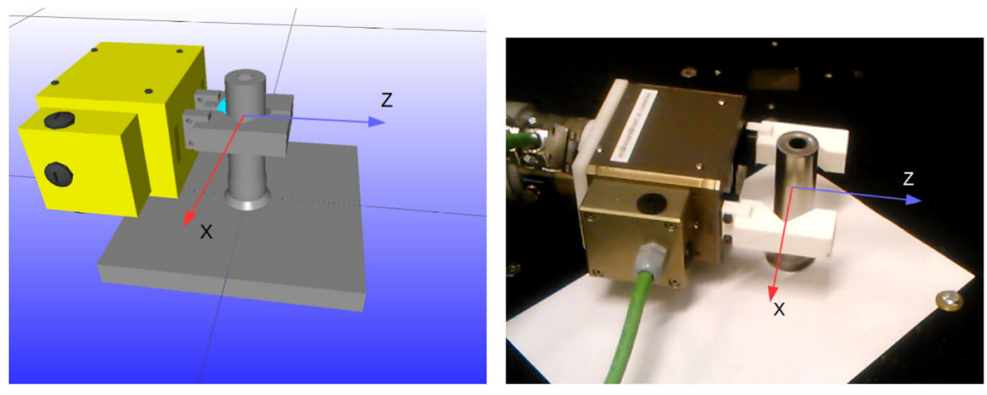

friction coefficient between the rotorcap object and the table $\left(\mu_{\text {table }}=0.26\right)$ and the friction coefficient for the printed fingers and the object ( $\mu_{\text {gripper }}=0.11$ ). The speed of the approaching robot was $v_{\text {robot }}=$ $0.24 \frac{\mathrm{m}}{\mathrm{s}}$ and the gripper closing force was $F_{\text {gripper }}=$ $30 \mathrm{~N}$.

Table 2 shows the ranges of the offsets applied to the gripper TCP frame in the experiments. Different kinds of sampling were applied in the simulated and in the real-world setting. Since simulation is much faster, a uniform dense sampling was used, with $\Delta_{\text {pos }}=$ $0.025 \mathrm{~mm}$ step for the position change and $\Delta_{\text {angle }}=$ $0.5^{\circ}$ step for the angle change. Since simulation is also deterministic, it was not necessary to repeat tests for each of the data points, as in the real-world setting (see the right column of Fig. 22, where the percentage of the succesful grasps is shown).

The grasps were classified according to their outcomes as either successful (with the object aligned after grasping) or as failures with different modes (collisions, object missed, dropped and grasped in incorrect alignment with the gripper). Figure 21 illustrates different grasp outcomes. In simulation, the grasp status was determined automatically, while in the real-world setting this was done through visual inspection by the operator.

For the sampling scheme, we have empirically found the ranges for which the change of the grasping mode occurs (i.e. when successes and failures are close together) and used a denser sampling in these areas (see Fig. 22). We have used sparser sampling in the areas in which no change of the outcome occurs. The real grasps were repeated 10 times for each of the data points in the densely sampled regions, and 3 times for the data points located in the sparse sampling regions.

The results in Fig. 22 show that the grasps are increasingly more likely to fail with increasing offsets. The obtained results show that the simulation is consistently more conservative in determining the
Table 2 The sampling of the grasp pose offsets tested in the simulation verification experiment

\begin{tabular}{|c|c|c|c|}
\hline & Range & Simulation sampling & Real-world sampling \\
\hline$x$ offset & $0 \mathrm{~mm} \leq x \leq 35 \mathrm{~mm}$ & $\Delta_{\mathrm{pos}}=0.025 \mathrm{~mm}$ & $\begin{array}{l}\text { Dense (in range }[27 \div 32.5 \mathrm{~mm}] \text { ): } \\
\Delta_{\text {pos }}=0.05 \mathrm{~mm} \\
\text { Sparse: } \Delta_{\text {pos }}=1 \mathrm{~mm}\end{array}$ \\
\hline$z$ offset & $0 \mathrm{~mm} \leq z \leq 25 \mathrm{~mm}$ & $\Delta_{\mathrm{pos}}=0.025 \mathrm{~mm}$ & $\begin{array}{l}\text { Dense (in range }[19 \div 23 \mathrm{~mm}] \text { ): } \\
\Delta_{\text {pos }}=0.05 \mathrm{~mm} \\
\text { Sparse: } \Delta_{\text {pos }}=1 \mathrm{~mm}\end{array}$ \\
\hline$R z$ (roll) offset & $0^{\circ} \leq R z \leq 60^{\circ}$ & $\Delta_{\text {angle }}=0.5^{\circ}$ & $\begin{array}{l}\left.\text { Dense (in range }\left[51 \div 59.5^{\circ}\right]\right): \\
\Delta_{\text {angle }}=0.5^{\circ} \\
\text { Sparse: } \Delta_{\text {angle }}=5^{\circ}\end{array}$ \\
\hline$R x$ (yaw) offset & $0^{\circ} \leq R x \leq 75^{\circ}$ & $\Delta_{\text {angle }}=0.5^{\circ}$ & $\begin{array}{l}\text { Dense (in range }\left[54 \div 74^{\circ}\right] \text { ): } \\
\Delta_{\text {angle }}=0.5^{\circ} \\
\text { Sparse: } \Delta_{\text {angle }}=5^{\circ}\end{array}$ \\
\hline
\end{tabular}



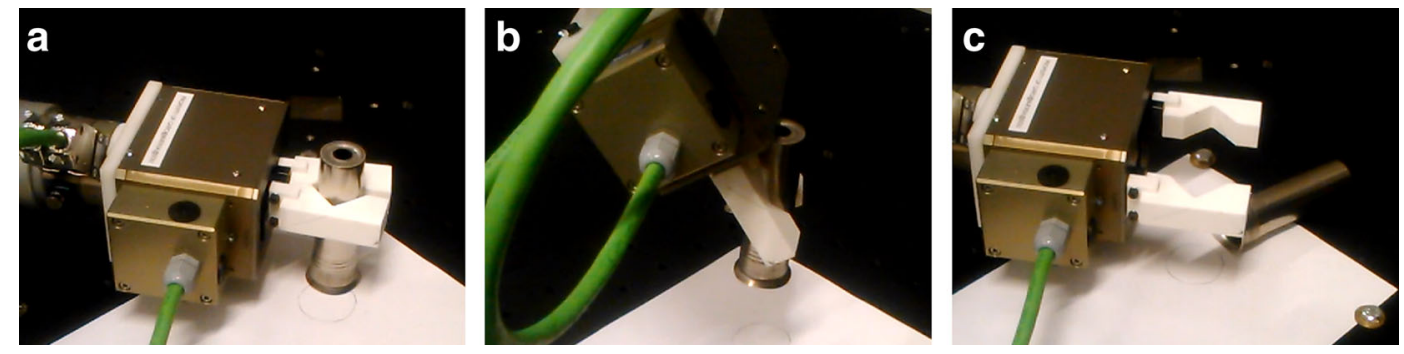

Fig. 21 Different grasp outcomes observed in the experiment: a) successful, b) failure with the object in incorrect alignment with the gripper, c) failure with the object is knocked over by the gripper due to a collision

successful grasp outcome as compared to the realworld results, as was also the tendency in experiments performed in [36]. This suggests that the gripper designs optimized using feedback from simulation are more robust to the uncertainties encountered in real grasping tasks. The slight difference between the simulated and real-world results is due to insufficient modelling of friction and of finger compliance, and also due to the quality of the finger printouts. Nevertheless, we can assert that the results obtained from grasp simulation qualitatively correspond to gripper performance observed in the real-world setting.

\subsection{Gripper Coverage Optimization}

In this experiment, we perform optimizations of the coverage index $C$ in the space of the general dimension parameters (1-length, 2-width, 3-depth, and 8TCP offset in Table 1) on the pump housing scene for the picking from all directions task (see Fig. 12). We selected these parameters as the most influential on the coverage index of a gripper finger. We chose the coverage optimization scenario for the value of the index in the context of bin picking - in which it is important to have many valid grasps covering the surface of the object.

We define the objective function for this experiment by picking the following weights:

$$
\begin{array}{rlll}
w_{S}=0 & w_{R}=0 & w_{A}=0 \quad & w_{C}=1 \\
w_{W}=0 & w_{\zeta}=0.01 \quad w_{V}=0.01
\end{array}
$$

As previously, the volume and stress indices ( $V$ and $\zeta$ respectively) weights were set to a low value of 0.01 , to make sure that the optimized gripper will still meet restrictions on its physical feasibility.

A number of grippers $G=100$ were heuristically generated within the selected parameters space defined by ranges presented in Table 1 . Similarly as in the previous experiment, the designs are based on the standard gripper design (see Fig. 14a), with length, width, depth, and TCP offset parameters changed. We have heuristically picked randomly sampled designs having quality indices that meet the following constraints: $C>0, \zeta>0$, and $V>0$. Three of these grippers were selected for further optimization. The gripper designs before, and after the procedure are presented in Fig. 23.

The results show that an improvement of the coverage index $C$ was achieved for all three gripper designs. The gripper length tends to increase slightly, while the TCP offset is placed at the very end of fingers. Moreover, the depth parameter of the fingers is reduced, which allows the gripper to grasp more fine features of the pump housing object. While the width of the finger also influences the coverage, it is not as critical, and needs to be increased to compensate for the decreased depth, in order to meet sufficiently low stress values. A good agreement in final design parameters can be found in cases pumpl_l1 and pumpl_l83.

The gripper finger design guidelines call for a reduction of the gripper footprint and to provide suitable clearance when approaching the object. Both of these objectives are achieved by the computed design, which shows a reduction in finger depth, and TCP placement at the finger tips, which offsets the base to avoid collisions with the objects or the fixture. Optimizing just the three parameters of the finger is a restrictive constraint, but nevertheless the produced design is sound from the engineering perspective. 
Fig. 22 The comparison of the results obtained from the simulated (on the left) and the real-world (on the right) experiments for grasps with offsets along in $x, z$, roll $(R z)$ and yaw $(R x)$. The bars show the percentage of the grasps with the success status (green) and failure status (red)
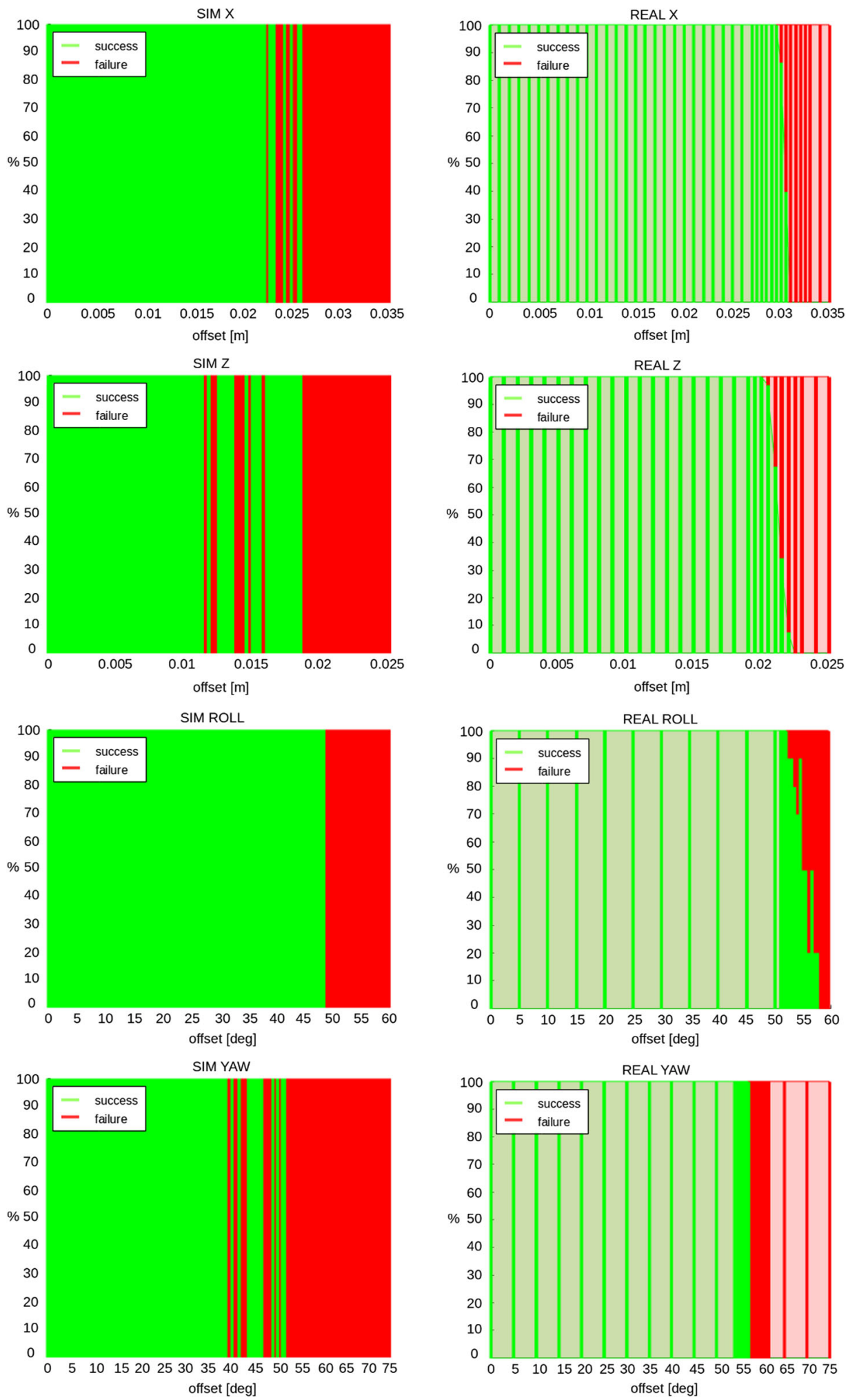
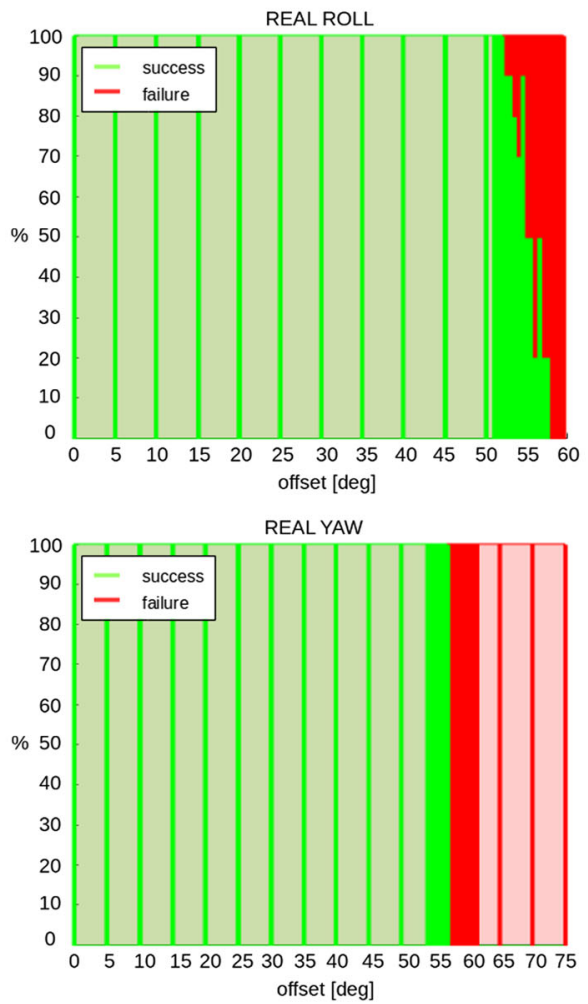


\begin{tabular}{|c|c|c|c|}
\hline & pump1_11 & pump1_49 & pump1_183 \\
\hline एँّ & $\begin{array}{l}\text { length: } 0.143 \\
\text { width: } 0.021 \\
\text { depth: } 0.023 \\
\text { TCP off.: } 1.91 \text {. } \\
10^{-3} \\
\text { C: } 2.06 \cdot 10^{-3} \\
\text { Q: } 2.29 \cdot 10^{-3}\end{array}$ & $\begin{array}{l}\text { length: } 0.122 \\
\text { width: } 0.020 \\
\text { depth: } 0.019 \\
\text { TCP off.: } 0.027 \\
\text { C: } 1.19 \cdot 10^{-5} \\
\text { Q: } 1.47 \cdot 10^{-5}\end{array}$ & $\begin{array}{l}\text { length: } 0.145 \\
\text { width: } 0.034 \\
\text { depth: } 8.18 \cdot 10^{-3} \\
\text { TCP off.: } 0.035 \\
\text { C: } 1.44 \cdot 10^{-4} \\
\text { Q: } 1.69 \cdot 10^{-4}\end{array}$ \\
\hline 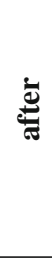 & $\begin{array}{l}\text { length: } 0.144 \\
\text { width: } 0.038 \\
\text { depth: } 4.00 \cdot 10^{-3} \\
\text { TCP off.: } 1.05 \text {. } \\
10^{-3} \\
\text { C: } 6.96 \cdot 10^{-3} \\
\text { Q: } 7.53 \cdot 10^{-3}\end{array}$ & $\begin{array}{l}\text { length: } 0.140 \\
\text { width: } 0.023 \\
\text { depth: } 9.81 \cdot 10^{-3} \\
\text { TCP off.: } 2.51 \cdot \\
10^{-3} \\
\text { C: } 6.00 \cdot 10^{-3} \\
\text { Q: } 6.50 \cdot 10^{-3}\end{array}$ & $\begin{array}{l}\text { length: } 0.151 \\
\text { width: } 0.040 \\
\text { depth: } 8.43 \cdot 10^{-3} \\
\text { TCP off.: } 4.14 \\
10^{-4} \\
\text { C: } 6.75 \cdot 10^{-3} \\
\text { Q: } 7.37 \cdot 10^{-3}\end{array}$ \\
\hline
\end{tabular}

Fig. 23 Gripper coverage optimization for the pump housing scene and picking from all directions task. Presented are the initial seed gripper designs (on the top) and the corresponding optimized designs (on the bottom)

\section{Conclusion}

A system for automatic computation of optimal gripper designs for a specific tasks and task contexts has been proposed in the paper. To optimize the design of a parametrized robot gripper - including both gripper parameters and parameters of the finger geometry a generic approach has been utilized. The method is based on dynamic simulation of the performance of a gripper in a virtual replica of the task context. To perform the simulation, the gripper parameters which influence the gripper functionality, i.e. stroke, motor force, shape of fingers, etc., as well as metrics for gripper quality evaluation, i.e. coverage, success ratio, wrench space measure and alignment were defined. For solving the optimization problem, we used the downhill Simplex method to optimize a weighted metric that combines the selected gripper quality metrics into a single quality function.

The experimental simulation part of the paper illustrates the capability of our optimization approach to arrive at reasonable gripper designs for different task contexts. We have shown that the introduced quality indices reflect the changes in finger geometry, including common design choices (e.g. chamfering, and cutouts). We have furthermore presented and explained the behaviour of the objective functions in the domain of selected design parameters. We have shown that certain aspects of the gripper performance can be improved by optimization within a selected subset of design parameters. We have demonstrated in our experiments the optimization of the gripper alignment index, and the coverage index. We provided a qualitative evaluation of the obtained results based on gripper design expertise. We have also compared the performance of the optimized gripper in both the simulation and in the real-world scenario, which showed a large degree of similarity in obtained results. By that we could confirm, that the proposed method allows for the computation of suitable gripper designs in simulation before they are manufactured.

In future work, we plan to compare and analyze, both the simulation results and in viva grasping experiments, which are planned to be performed in laboratory and production system environments. Further comparison of of results obtained for gripper designs tested in simulation and real-world environments is to be found in submitted works [36, 37]. We are aiming at arriving at a software solution for computation of gripper designs that can substitute the tedious design process with potentially many iterations of trial and error, which is usually applied nowadays when creating robotic assembly solutions.

Acknowledgments The research leading to these results has received funding from the European Community's Seventh Framework Programme FP7/2007-2013 (Programme and Theme: ICT-2011.2.1, Cognitive Systems and Robotics) under grant agreement no. 600578, ACAT and by Danish Agency for Science, Technology and Innovation, project CARMEN. 
Open Access This article is distributed under the terms of the Creative Commons Attribution 4.0 International License (http:// creativecommons.org/licenses/by/4.0/), which permits unrestricted use, distribution, and reproduction in any medium, provided you give appropriate credit to the original author(s) and the source, provide a link to the Creative Commons license, and indicate if changes were made.

\section{References}

1. Nelder, J.A., Mead, R.: A simplex method for function minimization. The Computer Journal 7(4), 308-313 (1965). [Online]. Available: http://comjnl.oxfordjournals. org/content/7/4/308.abstract

2. Wolniakowski, A., Miatliuk, K., Krüger, N., Rytz, J.A.: Automatic evaluation of task-focused parallel jaw gripper design. In: International Conference on Simulation, Modeling, and Programming for Autonomous Robots (2014)

3. Wolniakowski, A., Jorgensen, J.A., Miatliuk, K., Petersen, H.G., Krüger, N.: Task and context sensitive optimization of gripper design using dynamic grasp simulation. In: 20th International Conference on Methods and Models in Automation and Robotics (2015)

4. Causey, G.C., Quinn, R.D.: Gripper design guidelines for modular manufacturing. Proceedings of the 1998 IEEE International Conference on Robotics and Automation, 1998, vol. 2, pp. 1453-1458. IEEE (1998)

5. Causey, G.: Guidelines for the design of robotic gripping systems. Assem. Autom. 23(1), 18-28 (2003)

6. Krenich, S.: Multicriteria design optimization of robot gripper mechanisms, vol. 117, pp. 207-218. Springer, Netherlands (2004)

7. Boubekri, N., Chakraborty, P.: Robotic grasping: gripper designs, control methods and grasp configurations - a review of research. Integr. Manuf. Syst. 13(7), 520-531 (2002)

8. Blanes, C., Mellado, M., ortiz, C., Valera, A.: Review. technologies for robot grippers in pick and place operations for fresh fruits and vegetables. Span. J. Agric. Res. 9(4), 1130-1141 (2011)

9. Causey, G.C.: Elements of agility in manufacturing. Ph.D. dissertation Case Western Reserve University (1999)

10. Cuadrado, J., Naya, M.A., Ceccarelli, M., Carbone, G.: An optimum design procedure for two-finger grippers: a case of study. IFToMM Electronic Journal of Computational Kinematics 15403(1), 2002 (2002)

11. Lanni, C., Ceccarelli, M.: An optimization problem algorithm for kinematic design of mechanisms for two-finger grippers. Open Mechanical Engineering Journal 3, 49-62 (2009)

12. Cuadrado, J., Naya, M.A., Ceccarelli, M., Carbone, G.: An optimum design procedure for two-finger grippers: a case of study. IFToMM Electronic Journal of Computational Kinematics 15403(1), 2002 (2002)

13. Ceccarelli, M., Cuadrado, J., Dopico, D.: An optimum synthesis for gripping mechanisms by using natural coordinates. Proc. Inst. Mech. Eng. C J. Mech. Eng. Sci. 216(6), 643-653 (2002)

14. Zhang, T.: Optimal design of self-aligning robot gripper jaws. Ph.D. dissertation. aAI3044755 (2001)

15. Zhang, T., cheung, L., Goldberg, K.: Shape tolerance for robot gripper jaws. In: IROS, pp. 1782-1787 (2001)

16. Zhang, M.T., Goldberg, K.: Designing robot grippers: optimal edge contacts for part alignment. Robotica 25(03), 341 (2006)

17. Ellekilde, L.-P., Petersen, H.G.: Design and test of object aligning grippers for industrial applications. In: 2006 IEEE/RSJ International Conference on Intelligent Robots and Systems, pp. 5165-5170. IEEE (2006)

18. Datta, R., Deb, K.: Optimizing and deciphering design principles of robot gripper configurations using an evolutionary multi-objective optimization method (2011)

19. Kolluru, R., Valavanis, K., Smith, S., Tsourveloudis, N.: Design fundamentals of a reconfigurable robotic gripper system. IEEE Transactions on Systems, Man and Cybernetics, Part A: Systems and Humans 30(2), 181-187 (2000)

20. Song, D., Ek, C., Huebner, K., Kragic, D.: Multivariate discretization for bayesian network structure learning in robot grasping. In: 2011 IEEE International Conference on Robotics and Automation (ICRA), pp. 1944-1950 (2011)

21. Nikandrova, E., Kyrki, V.: Category-based task specific grasping. Robot. Auton. Syst. 70, 25-35 (2015)

22. Ciocarlie, M., Allen, P.: Data-driven optimization for underactuated robotic hands. In: 2010 IEEEInternational Conference on Robotics and Automation (ICRA), pp. 1292-1299. IEEE (2010)

23. Vassilis, M., Kostas, S., Stavros, P., Vassileios, S., Argiris, D., Nikos, A., et al.: Application of soft computing techniques in the design of robot grippers. Guidelines for a Decision Support Method Adapted to NPD Processes (2007)

24. Zhang, X., Nelson, C.A.: Multiple-criteria kinematic optimization for the design of spherical serial mechanisms using genetic algorithms. J. Mech. Des. 133(1), $011005-$ 011005 (2011)

25. Moulianitis, V.C., Aspragathos, N.A., Dentsoras, A.J.: A model for concept evaluation in design-an application to mechatronics design of robot grippers. Mechatronics 14(6), 599-622 (2004)

26. Kraft, D., Ellekilde, L.-P., Jorgensen, J.A.: Automatic grasp generation and improvement for industrial bin-picking. In: Röhrbein, F., Veiga, G., Natale, C. (eds.) Gearing $\mathrm{Up}$ and Accelerating Cross-fertilization between Academic and Industrial Robotics Research in Europe:, ser. Springer Tracts in Advanced Robotics, vol. 94, pp. 155176. Springer International Publishing, Cham (2014)

27. Krenich, S.: Optimal design of robot gripper mechanism using force and displacement transmission ratio. Applied Mechanics and Materials 613, 117-125 (2014)

28. Schunk: Schunk egrip. [Online]. Available: http://www. schunk-produkte.com/en/tools/3d-designtool-egrip.html (2015)

29. Rytz, J.A., Ellekilde, L.-P., Kraft, D., Petersen, H.G., Krüger, N.: On transferability of grasp-affordances in datadriven grasping. In: Proceedings of the RAAD 2013 22nd 
International Workshop on Robotics in Alpe-Adria-Danube Region (2013)

30. Jorgensen, J., Ellekilde, L., Petersen, H.: Robworksim an open simulator for sensor based grasping. In: Proceedings of Joint 41st International Symposium on Robotics (ISR 2010) and the 6th German Conference on Robotics (ROBOTIK 2010), pp. 1-8, Munich (2010)

31. Ferrari, C., Canny, J.: Planning optimal grasps. In: IEEE International Conference on Robotics and Automation (ICRA), pp. 2290-2295 (1992)

32. Zheng, Y., Qian, W.-H.: Improving grasp quality evaluation. Robot. Auton. Syst. 57, 665-673 (2009). [Online]. Available: http://www.sciencedirect.com/science/article/pii/S09 2188900800208X

33. Miller, A.T., Allen, P.K.: Graspit!: A versatile simulator for robotic grasping. IEEE Robot. Autom. Mag. 11, 110-122 (2004)

34. Kraft, D., Ellekilde, L.-P., Jorgensen, J.: Automatic grasp generation and improvement for industrial bin-picking, vol. 94, pp. 155-176. Springer International Publishing, Cham (2014)

35. Jorgensen, J.A., Rukavishnikova, N., Krüger, N., Petersen, H.G.: Spatial constraint identification of parts in se3 for action optimization. In: IEEE International Conference on Industrial Technology (ICIT) 03 (2015)

36. Wolniakowski, A., Gams, A., Kiforenko, L., Kramberger, A., Chrysostomou, D., Madsen, O., Miatliuk, K., Petersen, H.G., Hagelskjaer, F., Buch, A.G., Ude, A., Krüger, N.: Compensating pose uncertainties through appropriate gripper finger cutouts. submitted (2016)

37. Wolniakowski, A., Kramberger, A., Gams, A., Chrysostomou, D., Hagelskjaer, F., Thulesen, T.N., Kiforenko, L., Buch, A.G., Bodenhagen, L., Petersen, H.G., Madsen, O., Ude, A., Krüger, N.: Optimizing grippers for compensating pose uncertainties by dynamic simulation. In: International Conference on Simulation, Modeling, and Programming for Autonomous Robots (2016). submitted

Adam Wolniakowski is a Ph.D. student at the Automation and Robotics Department of the Białystok University of Technology. He received a M.Sc. degree in automation and robotics from Białystok University of Technology in 2011. His research interests include process simulation, machine learning and applied robotics.

Konstantsin Miatliuk is an associate professor of Białystok University of Technology, Poland. He received his M.Sc. degree in Robotics from the Belarusian State Technical University, Minsk, Belarus (1988), his Ph.D. degree in computer science from the Institute of Mathematics and Cybernetics, Minsk, Belarus (2003) and his Ph.D. degree in Automation and Robotics from AGH University of Science and Technology, Krakow, Poland (2006). He is IEEE member. His research interests include automation and robotics, systems science, computer science and mechatronics.
Zdzisław Gosiewski is a professor of the Białystok University of Technology, Poland. He is the head of the Automation and Robotics department. He received his M.Sc. degree from the Gdansk University of Technology, Poland (1974), his Ph.D. degree from the Institute of Fundamental Technological Research of the Polish Academy of Science (1981) and his Habilitation from Warsaw University of Technology, Poland (1990). His research interests include automation and robotics, mobile robots, UAV and mechatronics.

Leon Bodenhagen received the Master degree within Artificial Intelligence in 2009 and the Ph.D. degree in Robotics in 2013, both at Mærsk McKinney Møller Institute at the University of Southern Denmark. Since 2013 he has post doc position at the Mærsk McKinney Møller Institute. His main research interests cover the learning of robotic manipulation actions in industrial contexts and socially acceptable robot behaviors within domestic environments.

Henrik G. Petersen is Professor at the Maersk Mc-Kinney Moller Institute, University of Southern Denmark. He received his $\mathrm{PhD}$ degree from the Institute for Mathematics and Computer Science, Odense University in 1989. In 1996 he was employed at the Maersk McKinney Moller Institute, where he became professor in 2002 .

Lukas C. M. W. Schwartz received his B.Sc. in Robot Systems at the University of Southern Denmark in the year 2015. He is currently doing his M.Sc. in Robot Systems at the same university where he also is employed as a Student Programmer.

Jimmy A. Jørgensen obtained a M.Sc. degree in computer systems engineering at university of southern Denmark in 2006 and at the same place received his Ph.D. degree in robot systems engineering in 2010. He is currently Assistant Professor in the Mærsk Mc-Kinney Moller institute, University of Southern Denmark. Research interests include process simulation, dexterous grasping and robotics.

Lars-Peter Ellekilde is Associate Professor at the Maersk McKinney Moller Institute, University of Southern Denmark. He received his $\mathrm{PhD}$ degree from the Maersk Mc-Kinney Moller Institute in 2008 and have since been employed as assistant and associate professor focusing on robots for industrial applications.

Norbert Krüger is a professor at the Mærsk McKinney Møller Institute, University of Southern Denmark. He holds a M.Sc. degree from the Ruhr-Universität Bochum, Germany and his Ph.D. degree from the University of Bielefeld, Germany. His research covers computer vision, cognitive systems and applied robotics. 